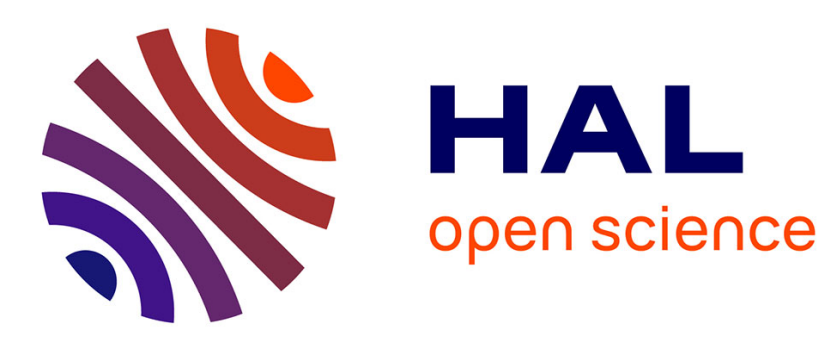

\title{
Automata on linear orderings
}

Véronique Bruyère, Olivier Carton

\section{- To cite this version:}

Véronique Bruyère, Olivier Carton. Automata on linear orderings. Journal of Computer and System

Sciences, 2007, 73 (1), pp.1-24. hal-00160925

\section{HAL Id: hal-00160925 \\ https://hal.science/hal-00160925}

Submitted on 9 Jul 2007

HAL is a multi-disciplinary open access archive for the deposit and dissemination of scientific research documents, whether they are published or not. The documents may come from teaching and research institutions in France or abroad, or from public or private research centers.
L'archive ouverte pluridisciplinaire HAL, est destinée au dépôt et à la diffusion de documents scientifiques de niveau recherche, publiés ou non, émanant des établissements d'enseignement et de recherche français ou étrangers, des laboratoires publics ou privés. 


\title{
Automata on linear orderings*
}

\author{
VÉRONIQUE BRUYÈre \\ Institut d'Informatique $^{\dagger}$ \\ Université de Mons-Hainaut
}

\author{
Olivier Carton \\ LIAFA $^{\ddagger}$ \\ Université Paris 7
}

September 25, 2006

\begin{abstract}
We consider words indexed by linear orderings. These extend finite, (bi-)infinite words and words on ordinals. We introduce finite automata and rational expressions for these words. We prove that for countable scattered linear orderings, these two notions are equivalent. This result extends Kleene's theorem.
\end{abstract}

\section{Introduction}

The theory of automata finds its origin in a paper of S. C. Kleene of 1956 where the basic theorem, known as Kleene's theorem, is proved for finite words [16]. Since then, the study of automata has become a branch of theoretical computer science in its own right and has developed in many directions, including study of automata working on infinite words, trees, and traces ([18, 24]).

In this paper we focus on automata working on linearly ordered objects. Examples of such objects are finite, infinite, bi-infinite and ordinal words, where the underlying linear ordering is respectively a finite ordering, the ordering of the positive integers, the ordering of the integers and the ordering of an ordinal. Each such class of words has its corresponding family of automata and in all cases, a Kleene-like theorem exists. Historically, Büchi introduced the so-called Büchi automata working on infinite words, to show the decidability of the monadic second order theory of $\langle\mathbb{N},<\rangle[8]$. He later extended the method to countable ordinals by using appropriate automata [9]. Büchi introduced $\omega$ rational operations for infinite words in [9]. For words indexed by an ordinal less than $\omega^{\omega}$, Choueka defined rational operations and proved an analogue of Kleene's theorem in [11]. This result is extended to any countable ordinal by Wojciechowski in [25]. The case of bi-infinite words is treated in [17, 13].

\footnotetext{
*partially supported by the CGRI-CNRS cooperation project Combinatoire et automates

${ }^{\dagger}$ Université de Mons-Hainaut, Le Pentagone, 6 avenue du Champ de Mars, B-7000 Mons, Belgium, Email: Veronique.Bruyere@umh.ac.be, Url: http://sun1.umh.ac.be/ vero/

${ }^{\ddagger}$ Université Paris 7 - case 7014, 2 place Jussieu, F-75251 Paris cedex 05, France, Email: Olivier.Carton@liafa.jussieu.fr, Url: http://www.liafa.jussieu.fr/ ${ }^{\sim}$ carton/
} 
The goal of this paper is to provide a unified approach of the study of words indexed by any countable linear ordering. We introduce a new notion of automaton which is simple, natural, and includes previously defined automata. We also define rational expressions and we prove the corresponding extension of Kleene's theorem (Theorem 20).

Words indexed by arbitrary countable linear orderings were first considered in [12] as solutions of systems of equations. They can be viewed as the frontiers of labeled binary trees in which the labels of the leaves are read from left to right. For these words, some kind of rational expressions have been studied in $[12,15,23]$. They have the nice property that the rational operations are total operators. For instance, an $\omega$-power can be concatenated with a finite word and the resulting word can be iterated by a reversed $\omega$-power. These operations lead to a characterization of the words which are the frontier of regular trees.

In our model, there are three groups of rational operations. First, there are the usual union, concatenation, finite iteration and omega iteration, as well as the ordinal iteration introduced in [25] for ordinal words. Next, there are reverse omega iteration and reverse ordinal iteration. They capture the leftinfinite ordinal words and the bi-infinite words. Finally, a last operation is necessary. It is the iteration for all linear orderings. This binary operation (denoted by $\diamond$ below) is subtle since it takes into account the cuts of a linear ordering as defined in [21].

We also define automata that work on words indexed by linear orderings. In our formulation, the notion of a path in an automaton depends heavily on cuts. In the case of a finite word $w$ of length $n$, the underlying ordering is $1<2<\cdots<n$. The $n+1$ states of a path for $w$ are inserted between the letters of $w$, i.e. at the cuts of the ordering. In general, for a word $w$ indexed by a linear ordering, the states of a path labeled by $w$ are indexed by the cuts of the ordering. Our automata have three types of transitions: the usual successor transitions, left limit transitions, and right limit transitions. For two consecutive states in a path, there is always a successor transition labeled by the letter in between. For a state $q$ which has no predecessor in a path, there is always a left limit transition between the left limit set $P$ and $q$. Right limit transitions are used when a state has no successor in a path. The notion of left limit set $P$ is already used in the acceptance condition of the classical Muller automata ([24]) which work on words of length $\omega$.

We think that our approach may have applications to the area of timed automata which are used for the specification and verification of real-time systems. Recently, ordinal words (called Zeno words) were considered as models of infinite sequences of actions which occur in a finite interval of time $[14,3]$. While the intervals of time are finite, infinite sequences of actions can be concatenated. A Kleene-like theorem already exists for standard timed automata (where infinite sequences of actions are supposed to generate divergent sequences of times) [1]. In [3], automata of Choueka and Wojciechowski are adapted to Zeno words. A kind of Kleene's theorem is proved, that is, the class of Zeno languages is the closure under an operation called refinement of the class of languages accepted by standard timed automata. 
The paper is organized as follows. Basic notions on linear orderings are given in Section 2. Words indexed by linear orderings are introduced in Section 3. Rational expressions denoting sets of such words are defined in Section 4. Automata accepting words on linear orderings are introduced in Section 5. Finally, Section 6 is devoted to the proof of the equivalence between rational expressions and automata.

Part of the results of the present paper has been presented at the conference MFCS'2001 [5].

\section{Linear orderings}

In this paper, we consider words on linear orderings, that is, totally ordered sequences of letters. We are only interested in countable linear orderings. Furthermore, we focus on scattered linear orderings. In this section, we provide the needed material on orderings. We recall the definitions and we fix the terminology. We refer the reader to [21] for a complete introduction to linear orderings.

A linear ordering $J$ is a set equipped with an ordering $<$ which is total, that is, for all $j \neq k$ in $J$, either $j<k$ or $k<j$ holds. The order type of an ordering $J$ is the class of all orderings isomorphic to $J$. The order types of $\mathbb{N}, \mathbb{Z}$ and $\mathbb{Q}$ under the usual ordering are respectively denoted by $\omega, \zeta$ and $\eta$. For every nonnegative integer $n$, the order type of an ordering with $n$ elements is denoted by $\mathbf{n}$. When we do not need to distinguish between isomorphic orderings, we will sometimes also use $\omega(\zeta, \eta, \mathbf{n})$ to stand for an arbitrary ordering of type $\omega$ (respectively, $\zeta, \eta, \mathbf{n}$ ).

Recall that an ordinal is a linear ordering which is well-ordered. This means that any non-empty subset has a least element. In this paper, we only consider countable ordinals, that is, ordinals less than the ordinal $\omega_{1}$.

Given a linear ordering $J$, we denote by $-J$ the backwards linear ordering obtained by reversing the ordering relation. Suppose that the ordering relation of $J$ is denoted by the symbol <. The ordering $-J$ has the same underlying set but its ordering relation $<^{*}$ is defined by $j<^{*} k$ if and only if $k<j$. For instance, the ordering $-\mathbb{N}$ is the ordering $\ldots, 3,2,1,0$. Its order type is denoted by $-\omega$.

Example 1 Let $\mathbb{Z}^{2}$ be the set of all pairs $(k, j)$ of integers. Define the relation $\left(k_{1}, j_{1}\right)<\left(k_{2}, j_{2}\right)$ by $j_{1}<j_{2}$ or $j_{1}=j_{2}$ and $k_{1}<k_{2}$. The relation $<$ linearly orders $\mathbb{Z}^{2}$.

Example 2 Let $\mathbb{Z}^{\omega}$ be the set of all sequences $\left(j_{n}\right)_{n \geq 0}$ of integers in which there are finitely many $n$ such that $j_{n} \neq 0$. Define the relation $\left(j_{n}\right)_{n \geq 0}<\left(k_{n}\right)_{n \geq 0}$ if and only if $j_{m}<k_{m}$ where $m$ is the greatest integer such that $j_{m} \neq k_{m}$. The relation $<$ endows $\mathbb{Z}^{\omega}$ with a linear ordering. It can be proved that if $m$ is taken as the least integer such that $j_{m} \neq k_{m}$, one obtains an ordering isomorphic to $\mathbb{Q}$.

Two elements $j$ and $k$ of a linear ordering $J$ are called consecutive if $j<k$ and if there is no element $i \in J$ such that $j<i<k$. The element $j$ is then called 
the predecessor of $k$ and $k$ is called the successor of $j$. Note that an element $k$ has a predecessor if and only if the set $\{j \mid j<k\}$ has a greatest element.

Let $K$ be a subset of a linear ordering $J$. If the least or the greatest element of $K$ exists, it is denoted by $\min (K)$ or $\max (K)$, and $K$ is then said to be left closed or right closed. Otherwise, it is said to be left open or right open. If the the greatest lower bound or the least upper bound of $K$ exists, it is denoted by $\inf (K)$ or $\sup (K)$. Note that if $K$ is left closed, its least element is also the greatest lower bound of $K$, that is, $\inf (K)=\min (K)$. An ordering $J$ is complete if any subset $K$ which is lower bounded has a greatest lower bound (or equivalently if any subset $K$ which is upper bounded has a least upper bound).

An ordering $K$ is a subordering of an ordering $J$ if $K$ is a subset of $J$ and if the order of $K$ is the restriction to $K$ of the order of $J$. An interval of a linear ordering $J$ is a subset $K$ of $J$ such that for all $j, k \in K$ and $i \in J$, the relation $j<i<k$ implies that $i$ belongs to $K$.

Let $J$ and $K$ be two linear orderings. The linear ordering $J+K$ is the ordering obtained by juxtaposition of $J$ and $K$. More formally, it is the linear ordering on the disjoint union $J \cup K$ extended with $j<k$ for all $j \in J$ and $k \in K$. For instance, if $J$ and $K$ are the orderings $-\mathbb{N}$ and $\mathbb{N}$, the ordering $J+K$ is isomorphic to $\mathbb{Z}$.

More generally, let $J$ be a linear ordering and let $K_{j}$ be a linear ordering for all $j \in J$. The linear ordering $\sum_{j \in J} K_{j}$ is the set of all pairs $(k, j)$ such that $k \in K_{j}$. The relation $\left(k_{1}, j_{1}\right)<\left(k_{2}, j_{2}\right)$ holds if and only if $j_{1}<j_{2}$ or $j_{1}=j_{2}$ and $k_{1}<k_{2}$ in $K_{j_{1}}$. For instance, if $J=\mathbb{Z}$ and $K_{j}=\mathbb{Z}$ for $j \in \mathbb{Z}$, the sum $\sum_{j \in J} K_{j}$ is isomorphic to the ordering $\mathbb{Z}^{2}$ already considered in Example 1.

\subsection{Cuts}

A Dedekind cut or simply a cut of a linear ordering $J$ is a pair $(K, L)$ of intervals such that $J=K \cup L$ and such that for all $k \in K$ and $l \in L, k<l$. Note that $K$ and $L$ are disjoint - in fact they form a partition of $J$. The set of all cuts of the ordering $J$ is denoted by $\hat{J}$.

Example 3 Let $J$ be the ordering $\{1,2,3\}$. The set $\hat{J}$ contains the four cuts $(\varnothing,\{1,2,3\}),(\{1\},\{2,3\}),(\{1,2\},\{3\})$ and $(\{1,2,3\}, \varnothing)$. More generally, if $J$ contains $n$ elements, $\hat{J}$ contains $n+1$ cuts.

The two trivial cuts $(\varnothing, J)$ and $(J, \varnothing)$ are usually not considered as cuts in the literature [21] but they are essential for our purpose and they are included in the set $\hat{J}$. However, it is sometimes convenient to ignore these two cuts and we denote by $\hat{J}^{*}$ the set $\hat{J}-\{(\varnothing, J),(J, \varnothing)\}$ of non-trivial cuts.

The set $\hat{J}$ can be linearly ordered as follows. For all cuts $c_{1}=\left(K_{1}, L_{1}\right)$ and $c_{2}=\left(K_{2}, L_{2}\right)$, define the relation $c_{1}<c_{2}$ if and only if $K_{1} \subsetneq K_{2}$. This inclusion implies $L_{1} \supsetneq L_{2}$ and the definition is therefore symmetric. The cuts $(\varnothing, J)$ and $(J, \varnothing)$ are the least and the greatest element of $\hat{J}$. They are respectively called the first and the last cut of $J$. We denote them by $c_{\min }$ and $c_{\max }$. 
Example 4 Let $J$ be the ordering $\mathbb{N}$. The set $\hat{J}$ contains the cut $(\{0, \ldots, n-$ $1\},\{n, n+1, \ldots\})$ for each integer $n \in \mathbb{N}$ and the last cut $(\mathbb{N}, \varnothing)$. The ordering $\hat{J}$ is thus isomorphic to the ordering $\mathbb{N}+1$ of order type $\omega+1$.

More generally, if $J$ is an ordinal $\alpha$, the ordering $\hat{J}$ is the ordinal $\alpha+1$. Indeed, for any cut $c=(K, L)$ different from the last one, the interval $L$ has a least element $j_{c}$ and the function which maps the cut $c$ to $j_{c}$ is one-to-one.

From the previous examples, it may seem that the ordering $\hat{J}$ is close to the ordering $J$. The following example shows that this is not always true.

Example 5 Let $J$ be the ordering $\mathbb{Q}$. The set $\hat{J}$ is not countable since each irrational number defines a unique cut of $\mathbb{Q}$.

More generally, if $J$ contains a subordering of order type $\eta$, then $\hat{J}$ is not countable. Therefore, we will only consider countable scattered linear orderings as defined in the next section.

For any $J$, the ordering $\hat{J}$ is complete and we often use this fact in the sequel. Indeed, the least upper bound of a subset $M$ of $\hat{J}$ is the cut $\left(K_{0}, L_{0}\right)$ where the intervals $K_{0}$ and $L_{0}$ are defined by

$$
K_{0}=\bigcup_{(K, L) \in M} K \quad \text { and } \quad L_{0}=\bigcap_{(K, L) \in M} L .
$$

The greatest lower bound of $M$ is given analogously.

\section{$2.2 \quad$ Scattered linear orderings}

A linear ordering $J$ is said to be dense if it contains at least two elements and if for all $i<k$ in $J$, there is $j \in J$ such that $i<j<k$. A linear ordering is scattered if it contains no dense subordering. Thus scattered orderings are those that do not contain a subordering of order type $\eta$. The following characterization of countable scattered linear orderings is due to Hausdorff.

Theorem 6 (Hausdorff) A countable linear ordering $J$ is scattered if and only if $J$ belongs to $\bigcup_{\alpha<\omega_{1}} V_{\alpha}$ where the classes $V_{\alpha}$ are inductively defined by

1. $V_{0}=\{J \mid J$ of order type $\mathbf{0}$ or $\mathbf{1}\}$;

2. $V_{\alpha}=\left\{\sum_{j \in J} K_{j} \mid J\right.$ of order type $\mathbf{n}, \omega,-\omega$, or $\zeta$ and $\left.K_{j} \in \bigcup_{\beta<\alpha} V_{\beta}\right\}$.

The orderings $\mathbb{Z}^{2}$ and $\mathbb{Z}^{\omega}$ considered in Examples 1 and 2 are scattered. They respectively belong to the classes $V_{2}$ and $V_{\omega}$ defined above. In the sequel, we denote by $\mathcal{S}$ the class of all countable scattered linear orderings. It follows from the previous theorem that if $J$ is a countable scattered linear ordering, then $\hat{J}$ is also a countable scattered linear ordering. Conversely, if $\hat{J}$ is countable, then $J$ is countable and scattered. 


\subsection{The ordering $J \cup \hat{J}$}

The orderings of $J$ and $\hat{J}$ can be extended to an ordering on the disjoint union $J \cup \hat{J}$ as follows. This means that $J \cup \hat{J}$ can be endowed with a linear ordering such that $J$ and $\hat{J}$ are then two of its suborderings. For $j \in J$ and a cut $c=(K, L)$, define the relations $j<c$ and $c<j$ by, respectively, $j \in K$ and $j \in L$. Note that exactly one of these two relations holds since $(K, L)$ is a partition of $J$. These relations together with the orderings of $J$ and $\hat{J}$ endow $J \cup \hat{J}$ with a linear ordering. This ordering will be used in Section 4. Notice that for any two consecutive elements $j_{1}<j_{2}$ of $J$, there is exactly one cut $c$ such that $j_{1}<c<j_{2}$. Analogously, for any two consecutive cuts $c_{1}<c_{2}$ of $\hat{J}$, there is exactly one element $j \in J$ such that $c_{1}<j<c_{2}$. The elements of $J$ and the cuts of $J$ interleave.

$$
|\cdots| \cdots|\bullet| \bullet|\bullet \cdots| \cdots\left|\underset{c_{j}^{-} c_{j}^{+}}{j}\right| \cdot|\cdot| \cdots|\bullet| \bullet|\bullet \cdots| \cdots \mid
$$

Figure 1: Ordering $J \cup \hat{J}$ for $J=\mathbb{Z}^{2}$

Example 7 The ordering $J=\mathbb{Z}^{2}$ is equal to the sum $\sum_{j \in \mathbb{Z}} \mathbb{Z}$ introduced in Example 1. There is a cut between each pair of consecutive elements in each copy of $\mathbb{Z}$, but there is also a cut between consecutive copies of $\mathbb{Z}$. There are also the first and the last cuts. The ordering $J \cup \hat{J}$ is pictured in Figure 1 where each element of $J$ is represented by a bullet and each cut by a vertical bar.

For each element $j \in J$, there are two consecutive cuts $c_{j}^{-}$and $c_{j}^{+}$such that $c_{j}^{-}<j<c_{j}^{+}$. They are defined as $c_{j}^{-}=(K,\{j\} \cup L)$ and $c_{j}^{+}=(K \cup\{j\}, L)$ with $K=\{k \mid k<j\}$ and $L=\{k \mid j<k\}$. Thus there is a one-to-one correspondence between elements of $J$ and pairs of consecutive cuts in $\hat{J}$. Notice that a cut $c=(K, L)$ has a predecessor if and only if $K$ has a greatest element $j$. In this case the cut $c$ is equal to $c_{j}^{+}$and its predecessor (in $\hat{J}$ ) is $c_{j}^{-}$. The cut $c=(K, L)$ has a successor if and only if $L$ has a least element $j$. It is then equal to $c_{j}^{-}$and its successor (in $\hat{J}$ ) is $c_{j}^{+}$.

The first and last cut of $J$ are respectively the least and the greatest element of the ordering $J \cup \hat{J}$. Thus the ordering $J \cup \hat{J}^{*}$ is the one obtained by removing the first and the last cut from $J \cup \hat{J}$. A straightforward induction on the rank of the classes $V_{\alpha}$ in Theorem 6 shows that if $J$ is a countable scattered ordering, then $J \cup \hat{J}$ and $J \cup \hat{J}^{*}$ are countable and scattered as well.

Both orderings $J \cup \hat{J}$ and $J \cup \hat{J}^{*}$ are complete. The following lemma gives a characterization of the ordering $J \cup \hat{J}$. It is needed in Section 6.2.

Lemma 8 Let $K$ be a complete scattered linear ordering with a least and a greatest element. Let $J$ and $J^{\prime}$ be two suborderings such that $J \cap J^{\prime}=\varnothing$ and $K=J \cup J^{\prime}$. Suppose that for consecutive elements $k$ and $k^{\prime}$ of $K$, either $k \in J$ 
and $k^{\prime} \in J^{\prime}$ or $k \in J^{\prime}$ and $k^{\prime} \in J$. Suppose also that any element of $J$ has a predecessor and a successor in $J^{\prime}$. Then $J^{\prime}$ is isomorphic to $\hat{J}$ and $K$ is isomorphic to $J \cup \hat{J}$.

Proof We define a function $f$ from $K$ into $J \cup \hat{J}$ as follows. For any $k \in K$, define

$$
f(k)= \begin{cases}k & \text { if } k \in J \\ (\{j \in J \mid j<k\},\{j \in J \mid k<j\}) & \text { if } k \in J^{\prime} .\end{cases}
$$

Since $J \cap J^{\prime}=\varnothing$ and $K=J \cup J^{\prime}$, the function $f$ is well defined. The restriction of $f$ to $J$ is the identity. The image of an element of $J^{\prime}$ is a cut of $J$. Therefore $f$ is a function from $K$ into $J \cup \hat{J}$.

We claim that the function $f$ is one-to-one. Thus we must show that $k \neq k^{\prime}$ implies $f(k) \neq f\left(k^{\prime}\right)$. If $k \in J$ or $k^{\prime} \in J$, the result is trivial. Suppose then that $k, k^{\prime} \in J^{\prime}$ and that $k<k^{\prime}$. Since $K$ is scattered, there are two consecutive elements $j<j^{\prime}$ of $K$ such that $k \leq j<j^{\prime} \leq k^{\prime}$. Since $j$ and $j^{\prime}$ are consecutive, either $j$ belongs to $J$ or $j^{\prime}$ belongs to $J$. Therefore the two cuts $f(k)$ and $f\left(k^{\prime}\right)$ are different.

We now prove that the function $f$ is onto. It is clear that $J \subseteq f(K)$. Let $(L, M)$ be a cut of $J$. We claim that there is $k \in J^{\prime}$ such that $(L, M)=f(k)$. Since $K$ is complete and has a least and a greatest element, any subset of $K$ has a greatest lower bound and a least upper bound. Define the two elements $l$ and $m$ of $K$ by $l=\sup (L)$ and $m=\inf (M)$. If $l$ belongs to $L$, it has a successor $k$ in $J^{\prime}$ and one has $(L, M)=f(k)$. If $m$ belongs to $M$, it has a predecessor $k$ in $J^{\prime}$ and one has $(L, M)=f(k)$. If $l$ and $m$ do not belong to $L$ and $M$, they belong to $J^{\prime}$ and their image by $f$ is the cut $(L, M)$.

\subsection{Condensation}

The following notion is needed in Section 6.2. Let $J$ be a linear ordering. A condensation of $J$ is an equivalence relation $\sim$ on $J$ such that each of its classes is an interval. The ordering of $J$ induces a linear ordering of the quotient $J / \sim$.

The quotient $J / \sim$ inherits some properties of $J$. If $J$ has a least or a greatest element, then $J / \sim$ also has a least or a greatest element which is the class of the least or greatest element of $J$. If $J$ is complete, then $J / \sim$ is also complete and if $J$ is scattered, then $J / \sim$ is also scattered.

We mention here some useful properties of consecutive classes $k$ and $k^{\prime}$ of $J / \sim$ when the ordering $J$ is complete. Define $j$ and $j^{\prime}$ by $j=\sup (k)$ and $j^{\prime}=\inf \left(k^{\prime}\right)$. By definition, one has $j \leq j^{\prime}$. If $j<j^{\prime}$, then $j$ and $j^{\prime}$ respectively belong to $k$ and $k^{\prime}$ since the classes $k$ and $k^{\prime}$ are consecutive. In that case, the interval $k$ is right closed and $k^{\prime}$ is left closed. If $j=j^{\prime}$, then $j$ belongs to either $k$ or $k^{\prime}$. In the former case, $k$ is right closed and $k^{\prime}$ is left open and in the latter case, $k$ is right open and $k^{\prime}$ is left closed. Note that it is impossible that $k$ is right open and $k^{\prime}$ is left open.

In the sequel, we consider only linear orderings which are countable and scattered. This restriction is needed in the proof of the main result (Theorem 20) 
which states that rational expressions and automata are equivalent. The definition of the rational operations given in Section 4 also depends on this restriction. The ordinal iterations in particular are restricted to countable ordinals. However, the definition of automata that we introduce in Section 5 makes sense for all linear orderings.

\section{Words on linear orderings}

Let $A$ be a finite alphabet whose elements are called letters. For a linear ordering $J$, a word of length $J$ over $A$ is a function which maps each element of $J$ to a letter of $A$. A word on a linear ordering $J$ can be viewed as a labeled ordering where each point of $J$ has been decorated by a letter. A word is denoted in a sequence-like notation by $\left(a_{j}\right)_{j \in J}$ where $a_{j} \in A$ is the image of the element $j$. The word whose length is the empty set is called the empty word and it is denoted by $\varepsilon$.

The notion of word just introduced generalizes notions common in the literature. If the ordering $J$ is finite with $n$ elements, a word of length $J$ is a finite sequence $a_{1} \ldots a_{n}$ [18]. A word of length $\mathbb{N}$ is a sequence $a_{0} a_{1} a_{2} \ldots$ which is usually called an $\omega$-word or an infinite word [24]. A word of length $\mathbb{Z}$ is a sequence $\ldots a_{-2} a_{-1} a_{0} a_{1} a_{2} \ldots$ which is usually called a bi-infinite word. An ordinal word is a word indexed by a countable ordinal.

Example 9 Recall that the ordering $\mathbb{N}+(-\mathbb{N})$ is the ordering of $\{(n, 0) \mid n \geq$ $0\} \cup\{(-n, 1) \mid n \geq 0\}$ with $\left(k_{1}, j_{1}\right)<\left(k_{2}, j_{2}\right)$ if and only if $j_{1}<j_{2}$ or $j_{1}=j_{2}$ and $k_{1}<k_{2}$. The word $x=b^{\omega}(a b)^{-\omega}$ is the word of length $\mathbb{N}+(-\mathbb{N})$ defined by

$$
x_{k, j}= \begin{cases}a & \text { if } j=1 \text { and } k \text { odd } \\ b & \text { otherwise }\end{cases}
$$

Let $x=\left(a_{j}\right)_{j \in J}$ and $y=\left(b_{k}\right)_{k \in K}$ be two words of length $J$ and $K$. The product (or the concatenation) of $x$ and $y$ is the word $z=\left(c_{l}\right)_{l \in J+K}$ of length $J+K$ such that

$$
c_{l}= \begin{cases}a_{l} & \text { if } l \in J \\ b_{l} & \text { if } l \in K\end{cases}
$$

The product of $x$ and $y$ is denoted $x y$. More generally, let $J$ be a linear ordering and for each $j \in J$, let $x_{j}$ be a word of length $K_{j}$. The product $\prod_{j \in J} x_{j}$ is the word $z$ of length $K=\sum_{j \in J} K_{j}$ defined as follows. Suppose that each word $x_{j}$ is equal to $\left(a_{k, j}\right)_{k \in K_{j}}$ and recall that $K$ is the set of all pairs $(k, j)$ such that $k \in K_{j}$. The product $z$ is then equal to $\left(a_{k, j}\right)_{(k, j) \in K}$. This definition can be extended to sets. Given sets $\left(X_{j}\right)_{j \in J}$, the product $\Pi_{j \in J} X_{j}$ is the set $\left\{\Pi_{j \in J} x_{j} \mid x_{j} \in X_{j}\right\}$. When $J$ is finite or isomorphic to $\omega$, the product is called a finite product or an $\omega$-product.

Two words $x=\left(a_{j}\right)_{j \in J}$ and $y=\left(b_{k}\right)_{k \in K}$ of length $J$ and $K$ are isomorphic if there is an order-preserving isomorphism $f$ from $J$ to $K$ such that $a_{j}=b_{f(j)}$ 
for all $j$ in $J$. This obviously defines an equivalence relation on words. A class of words up to isomorphism is called a type. In this paper, we identify isomorphic words. This makes sense since the automata and rational expressions we introduce do not distinguish isomorphic words: an automaton $\mathcal{A}$ accepting a word $x$, for example, also accepts any word isomorphic to $x$. Since isomorphic words are identified, the length of word is more an order type than an ordering. For instance, a word of length $\mathbb{N}$ (or $\mathbb{Z}$ ), is rather called a word of length $\omega$ (or $\zeta)$.

Note that some orderings, such as $\mathbb{Z}$, have non trivial internal isomorphisms. This induces some unexpected isomorphisms between words. For instance, let $x$ and $y$ be the words $\left(a_{j}\right)_{j \in \mathbb{Z}}$ and $\left(b_{j}\right)_{j \in \mathbb{Z}}$ defined by

$$
a_{j}=\left\{\begin{array}{ll}
a & \text { if } j=0 \\
b & \text { otherwise }
\end{array} \quad \text { and } \quad b_{j}= \begin{cases}a & \text { if } j=1 \\
b & \text { otherwise }\end{cases}\right.
$$

The words $x$ and $y$ are isomorphic since the function $f$ given by $f(x)=x+1$ is an automorphism of $\mathbb{Z}$. Using notation of Example 9, both words can be denoted $b^{-\omega} a b^{\omega}$.

Example 10 For $j \in \mathbb{Z}$, define the word $x_{j}$ by $x_{j}=b^{-\omega}$ if $j$ is even and by $x_{j}=a b^{\omega}$ if $j$ is odd. The product $\prod_{j \in J} x_{j}$ is the word $\left(b^{-\omega} a b^{\omega}\right)^{\zeta}$ of length $\zeta^{2}$.

\section{Rational expressions}

In this section, the rational operations used to define rational sets of words on scattered linear orderings are introduced. These rational operations include the usual Kleene operations for finite words - union, concatenation, and finite iteration (though these must be appropriately extended to our notion of word). They also include omega iteration (usually used to construct $\omega$-words) and the ordinal iteration introduced by Wojciechowski [25] for ordinal words. Three new operations are also needed: backwards omega iteration, backwards ordinal iteration, and an additional binary operation, a kind of general iteration for scattered linear orderings.

Union, concatenation and finite iteration are denoted as usual by the symbols ,$+ \cdot$ and $*$. Omega iteration and ordinal iteration are denoted by the symbols $\omega$ and $\sharp$ whereas backwards omega iteration and backwards ordinal iteration are denoted by the symbols $-\omega$ and $-\sharp$. Iteration for all scattered linear orderings is denoted by the symbol $\diamond$.

We first define the various sorts of iteration in a unified framework. Given a set $X$ of words and a class $\mathcal{J}$ of linear orderings, define iteration $X^{\mathcal{J}}$ of $X$ with respect to $\mathcal{J}$ by

$$
X^{\mathcal{J}}=\left\{\prod_{j \in J} x_{j} \mid J \in \mathcal{J} \text { and } x_{j} \in X\right\} .
$$

The sets $X^{*}, X^{\omega}, X^{-\omega}, X^{\sharp}$ and $X^{-\sharp}$ are then respectively equal to $X^{\mathcal{J}}$ for $\mathcal{J}$ equal to the class of all finite linear orderings, the class $\{\omega\}$ which only contains 
the ordering $\omega$, the class $\{-\omega\}$ which only contains the ordering $-\omega$, the class of all countable ordinals and the class $\left\{-\alpha \mid \alpha<\omega_{1}\right\}$ of all countable backwards ordinals.

We now define the binary operations. Let $X$ and $Y$ be two sets of words. The sets $X+Y, X \cdot Y$ and $X \diamond Y$ are defined by

$$
\begin{gathered}
X+Y=X \cup Y, \quad X \cdot Y=\{x y \mid x \in X \text { and } y \in Y\} \quad \text { and } \\
X \diamond Y=\left\{\prod_{j \in J \cup \hat{J}^{*}} z_{j} \mid J \in \mathcal{S} \backslash\{0\} \text { and } z_{j} \in X \text { if } j \in J \text { and } z_{j} \in Y \text { if } j \in \hat{J}^{*}\right\} .
\end{gathered}
$$

A word $x$ belongs to $X \diamond Y$ if and only if there is a non-empty countable scattered linear ordering $J$ such that $x$ is the product of a sequence of length $J \cup \hat{J}^{*}$ of words where each word indexed by an element of $J$ belongs to $X$ and each word indexed by a cut in $\hat{J}^{*}$ belongs to $Y$. Note that if the empty word $\varepsilon$ does not belong to $X$, it does not belong to $X \diamond Y$.

A rational expression over $A$ is a well-formed term of the free algebra over $\{\varnothing\} \cup A$ with the symbols denoting the rational operations as function symbols. We inductively define a mapping $L$ from this algebra into the family of sets of words over $A$ in the following way.

$$
\begin{aligned}
L(\varnothing) & =\varnothing, & L(a) & =\{a\}, \\
L\left(E^{*}\right) & =L(E)^{*}, & & \\
L\left(E^{\omega}\right) & =L(E)^{\omega}, & L\left(E^{-\omega}\right) & =L(E)^{-\omega}, \\
L\left(E^{\sharp}\right) & =L(E)^{\sharp}, & L\left(E^{-\sharp}\right) & =L(E)^{-\sharp}, \\
L(E+F) & =L(E)+L(F), & L(E \cdot F) & =L(E) \cdot L(F), \\
L(E \diamond F) & =L(E) \diamond L(F) . & &
\end{aligned}
$$

We say that the rational expression $E$ denotes the set $L(E)$. A set of words is rational if it can be denoted by a rational expression.

As usual, the dot denoting concatenation is omitted in rational expressions. Thus, the rational expression $a \cdot\left(a+a \cdot b^{\omega}\right)^{\sharp}$ is written $a\left(a+a b^{\omega}\right)^{\sharp}$. We also use the following abbreviations. If $E$ is a rational expression, the expressions $\varepsilon, E^{\zeta}$ and $E^{\diamond}$ respectively abbreviate $\varnothing^{*}, E^{-\omega} E^{\omega}$ and $E \diamond \varepsilon$. If the alphabet $A$ is the set $\left\{a_{1}, \ldots, a_{n}\right\}$, the symbol $A$ is also used as an abbreviation for the expression $a_{1}+\cdots+a_{n}$.

Example 11 The expressions $A^{\diamond}+\varepsilon$ and $\left(A^{\diamond}+\varepsilon\right) a\left(A^{\diamond}+\varepsilon\right)$ denote respectively the set of all words and the set of words having an occurrence of the letter $a$.

Example 12 The expression $A^{*}$ denotes the set of finite words and the expression $\left(A^{\diamond}\right)^{\omega}\left(A^{\diamond}+\varepsilon\right)+\left(A^{\diamond}+\varepsilon\right)\left(A^{\diamond}\right)^{-\omega}$ denotes its complement. Indeed, a linear ordering $J$ is not finite if and only if it has some cut $(K, L)$ such that either $K$ does not have a greatest element or $L$ does not have a least element. The rational expression $\left(A^{\diamond}\right)^{\omega}$ denotes the set of words whose length does not have a last element. Therefore, the expression $\left(A^{\diamond}\right)^{\omega}\left(A^{\diamond}+\varepsilon\right)$ denotes the set 
whose length has a cut $(K, L)$ such that $K$ does not have a greatest element. Symmetrically, the expression $\left(A^{\diamond}+\varepsilon\right)\left(A^{\diamond}\right)^{-\omega}$ denotes the set of words whose length has a cut $(K, L)$ such that $L$ does not have a least element.

In the sequel, we will often use the following lemma.

Lemma 13 The class of rational sets over an alphabet $A$ is closed under the rational operations.

Proof It is clear that if the sets $X$ and $Y$ are respectively denoted by the expressions $E$ and $F$, then the sets $X+Y, X \cdot Y, X^{*}, X^{\omega}, X^{-\omega}, X^{\sharp}, X^{-\sharp}$ and $X \diamond Y$ are respectively denoted by the expressions $E+F, E \cdot F, E^{*}, E^{\omega}, E^{-\omega}$, $E^{\sharp}, E^{-\sharp}$ and $E \diamond F$.

We say that a set $X$ has a rational expression over the sets $X_{i}$ if we can find an expression for $X$ in terms of the sets $X_{i}$ using only the rational operations. The previous lemma states that if $X$ has a rational expression over the sets $X_{i}$ and each set $X_{i}$ is rational, then $X$ is itself rational.

In the sequel, we also need the following lemma. Let $X_{1}, \ldots, X_{n}$ be sets of words over an alphabet $A$, and let $B$ be the alphabet $\left\{b_{1}, \ldots, b_{n}\right\}$. We define a function $\lambda$ which maps each letter $b_{i}$ to the set $X_{i}$. The function is first extended to each word $w=\left(b_{i_{j}}\right)_{j \in J}$ over $B$ by setting

$$
\lambda(w)=\prod_{j \in J} X_{i_{j}} .
$$

Second, it is extended to sets of words over $B$ by setting

$$
\lambda(W)=\bigcup_{w \in W} \lambda(w)
$$

Lemma 14 If each set $X_{i}$ is rational and if the set $W$ is rational, then the set $\lambda(W)$ is also rational.

Proof The proof is divided into two parts. In the first part, we show that $\lambda$ commutes with all rational operations. In the second part, we prove the statement of the lemma.

We begin by establishing the following equalities for any index set $I$ and any linear ordering $J$

$$
\begin{aligned}
\lambda\left(\bigcup_{i \in I} W_{i}\right) & =\bigcup_{i \in I} \lambda\left(W_{i}\right) \\
\lambda\left(\prod_{j \in J} W_{j}\right) & =\prod_{j \in J} \lambda\left(W_{j}\right) .
\end{aligned}
$$

Equality (1) follows immediately from the definition of $\lambda$. To prove Equality (2) we use the equality $\lambda\left(\prod_{j \in J} w_{j}\right)=\prod_{j \in J} \lambda\left(w_{j}\right)$ for any words $w_{j}$ which follows 
easily from the definition of $\lambda$.

$$
\begin{aligned}
\lambda\left(\prod_{j \in J} W_{j}\right) & =\lambda\left(\left\{\prod_{j \in J} w_{j} \mid w_{j} \in W_{j}\right\}\right) \\
& =\bigcup_{w_{j} \in W_{j}} \lambda\left(\prod_{j \in J} w_{j}\right) \\
& =\bigcup_{w_{j} \in W_{j}} \prod_{j \in J} \lambda\left(w_{j}\right) \\
& =\bigcup_{w_{j} \in W_{j}}\left\{\prod_{j \in J} z_{j} \mid z_{j} \in \lambda\left(w_{j}\right)\right\} \\
& =\prod_{j \in J} \lambda\left(W_{j}\right) .
\end{aligned}
$$

Two particular cases of Equalities (1) and (2) are $\lambda\left(W_{1}+W_{2}\right)=\lambda\left(W_{1}\right)+\lambda\left(W_{2}\right)$ and $\lambda\left(W_{1} \cdot W_{2}\right)=\lambda\left(W_{1}\right) \cdot \lambda\left(W_{2}\right)$.

The following computation shows that $\lambda$ commutes with the finite iteration.

$$
\lambda\left(W^{*}\right)=\lambda\left(\bigcup_{n \geq 0} W^{n}\right)=\bigcup_{n \geq 0} \lambda\left(W^{n}\right)=\bigcup_{n \geq 0} \lambda(W)^{n}=\lambda(W)^{*} .
$$

Similar computations show that $\lambda$ commutes with the rational operations $\omega$, $-\omega, \sharp$, and $-\sharp$. It remains to prove that $\lambda\left(W_{1} \diamond W_{2}\right)=\lambda\left(W_{1}\right) \diamond \lambda\left(W_{2}\right)$, an equality which is established as for Equality (2).

The statement of the lemma is proved by induction on the structure of the rational expression denoting the set $W$. The base cases $\left(W=\varnothing\right.$ and $\left.W=\left\{b_{i}\right\}\right)$ follow from the definition of $\lambda$ and the hypothesis that $X_{i}$ is rational. The induction step easily follows from the first part of the proof and Lemma 13.

In the rest of the paper, we often make no distinction between a rational expression $E$ and the set $L(E)$ denoted by it. For instance, we write $a\left(a+a b^{\omega}\right)^{\sharp}$ for the set $L\left(a\left(a+a b^{\omega}\right)^{\sharp}\right)$.

\section{Automata}

In this section, automata on words on linear orderings are defined. As in the rest of the paper, linear orderings considered in this section are countable and scattered. However, it is worth pointing out that our definition of automata is suitable for all linear orderings. Automata that we define are a natural generalization of Büchi automata [9] on ordinal words. Automata introduced by Büchi are usual (Kleene) automata with left limit transitions of the form $P \rightarrow p$ used for limit ordinals. The automata that we introduce have limit transitions of the form $p \rightarrow P$ as well. 
Definition 15 Let $A$ be a finite alphabet. An automaton $\mathcal{A}$ over $A$ is a 4-tuple $(Q, E, I, F)$ where $Q$ is a finite set of states, $E \subseteq(Q \times A \times Q) \cup(\mathcal{P}(Q) \times Q) \cup$ $(Q \times \mathcal{P}(Q))$ is the set of transitions, $I \subseteq Q$ is the set of initial states and $F \subseteq Q$ is the set of final states.

Since the alphabet and the set of states are finite, the set of transitions is also finite. Transitions are either of the form $(p, a, q)$ or of the form $(P, q)$ or of the form $(q, P)$ where $P$ is a subset of $Q$. A transition of the former case is called a successor transition and it is denoted by $p \stackrel{a}{\rightarrow} q$. A transition of the two latter cases are respectively called a left limit or a right limit transition and they are denoted by $P \rightarrow q$ and $q \rightarrow P$. Before explaining what a path is and how an automaton accepts words, we illustrate this definition with the following example.
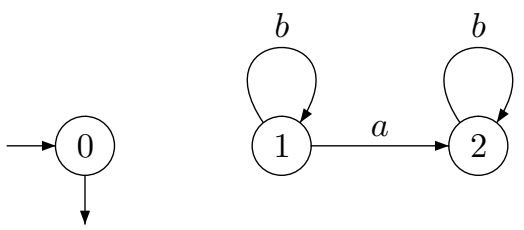

$$
\begin{aligned}
& 0 \rightarrow\{1\} \\
& \{2\} \rightarrow 0
\end{aligned}
$$

Figure 2: Automaton of Example 16

Example 16 The automaton pictured in Figure 2 has 3 successor transitions which are pictured as labeled edges of a graph. It also has a left limit transition $\{2\} \rightarrow 0$ and a right limit transition $0 \rightarrow\{1\}$. The state 0 is the only initial state and the only final state.

We say that a transition leaves a state $q$ if it is either a successor transition $q \stackrel{a}{\rightarrow} p$ for some state $p$ or a right limit transition $q \rightarrow P$ for some subset $P$ of states. We say that it enters a state $q$ if it is either a successor transition $p \stackrel{a}{\rightarrow} q$ or a left limit transition $P \rightarrow q$. The sets of transitions leaving and entering a state $q$ are respectively denoted by $\operatorname{Out}(q)$ and $\operatorname{In}(q)$. More generally, for a set $P$ of states, we define $\operatorname{Out}(P)=\bigcup_{q \in P} \operatorname{Out}(q)$ and $\operatorname{In}(P)=\bigcup_{q \in P} \operatorname{In}(q)$.

In order to define the notion of path in such an automaton, the following notion of limits is needed. We define it for an arbitrary linear ordering $J$ but we use it when the considered ordering is actually the ordering $\hat{J}$ of cuts of a given ordering $J$. Let $Q$ be a finite set, let $J$ be a linear ordering and let $\gamma=\left(q_{j}\right)_{j \in J}$ be a word over $Q$. Let $j$ be a fixed element of $J$. The left limit set and right limit set of $\gamma$ at $j$ are the two subsets $\lim _{j^{-}} \gamma$ and $\lim _{j^{+}} \gamma$ of $Q$ defined as follows.

$$
\begin{array}{ll}
\lim _{j^{-}} \gamma=\{q \in Q \mid \forall k<j & \left.\exists i k<i<j \text { and } q=q_{i}\right\}, \\
\lim _{j^{+}} \gamma=\{q \in Q \mid \forall k>j & \left.\exists i j<i<k \text { and } q=q_{i}\right\} .
\end{array}
$$

Thus the left limit at $j$ is the set of states to the left that occur infinitely often infinitely close to $j$. Note that if $j$ has a predecessor, the $\operatorname{limit}$ set $\lim _{j^{-}} \gamma$ is 
empty. Conversely, if $j$ has no predecessor and is not the least element of $J$, $\lim _{j^{-}} \gamma$ is non-empty since the set $Q$ is finite. Similar results hold for right limit sets. For $c=\min (J)$, we define $\lim _{c^{-}} \gamma=\varnothing$ and for $c=\max (J)$, we define $\lim _{c^{+}} \gamma=\varnothing$ (although these two limits are literally defined as equal to $Q$ by the above formulas).

We now come to the definition of a path in an automaton on linear orderings. Roughly speaking, a path is a labeling of each cut of the ordering by a state of the automaton such that local properties are satisfied.

Definition 17 Let $\mathcal{A}$ be an automaton and let $x=\left(a_{j}\right)_{j \in J}$ be a word of length $J$. A path $\gamma$ labeled by $x$ is a sequence of states $\gamma=\left(q_{c}\right)_{c \in \hat{J}}$ of length $\hat{J}$ such that

- For consecutive cuts $c_{j}^{-}$and $c_{j}^{+}, q_{c_{j}^{-}} \stackrel{a_{j}}{\longrightarrow} q_{c_{j}^{+}}$is a successor transition.

- For any cut $c$ which is not the first cut and which has no predecessor, $\lim _{c^{-}} \gamma \rightarrow q_{c}$ is a left limit transition.

- For each cut $c$ which is not the last cut and which has no successor, $q_{c} \rightarrow$ $\lim _{c^{+}} \gamma$ is a right limit transition.

It is worth pointing out that the length of a path labeled by a word $x$ of length $J$ is the ordering $\hat{J}$ of the cuts of $J$. Since a sequence of states indexed by $\hat{J}$ is actually a function from $\hat{J}$ into $Q$, we sometimes use a functional notation and the state $q_{c}$ of a path $\gamma$ is also denoted by $\gamma(c)$.

By the previous definition, there is a transition entering the state $\gamma(c)$ for each cut $c$ which is not the first cut. This transition is a successor transition if the cut $c$ has a predecessor in $\hat{J}$ and it is a left limit transition otherwise. Similarly, there is a transition leaving $\gamma(c)$ for any cut $c$ which is not the last cut.

Since the ordering $\hat{J}$ has a least and a greatest element, a path always has a first and a last state, which are the images of the first and the last cut. A path is successful if its first state is initial and its last state is final. A word is accepted or recognized by the automaton if it is the label of a successful path. A set of words is recognizable if it is the set of words accepted by some automaton.

The notion of path we have introduced for words on orderings coincides with the usual notion of paths considered in the literature for finite words, $\omega$-words and ordinal words. Let $x$ be a finite word $a_{1} \ldots a_{n}$. The set of cuts of the finite ordering $\{1, \ldots, n\}$ can be identified with $\{1, \ldots, n+1\}$ (see Example 3 ). In our setting, a path labeled by $x$ is then a finite sequence $q_{1}, \ldots, q_{n+1}$ of states such that $q_{j} \stackrel{a_{j}}{\longrightarrow} q_{j+1}$ is a successor transition for each $j$ in $\{1, \ldots, n\}$. This matches the usual definition of a finite path in an automaton [18, p. 5].

Let $x=a_{0} a_{1} a_{2} \ldots$ be an $\omega$-word. The set of cuts of the ordering $J=\omega$ is the ordinal $\omega+1=\{0,1,2, \ldots, \omega\}$ (see Example 4). The pairs of consecutive cuts are the pairs $(j, j+1)$ for $j<\omega$ whereas the cut $c=\omega$ has no predecessor. In our setting, a path $\gamma$ labeled by $x$ is a sequence $q_{0}, q_{1}, q_{2}, \ldots, q_{\omega}$ of states such that $q_{j} \stackrel{a_{j}}{\longrightarrow} q_{j+1}$ is a successor transition for all $j<\omega$ and such that $\lim _{\omega^{-}} \gamma \rightarrow q_{\omega}$ is a left limit transition. Note that $\lim _{\omega^{-}} \gamma$ is the set of states 
which occur infinitely many times in $\gamma$. This path is successful if and only if $q_{0}$ is initial and $q_{\omega}$ is final. Define the family $\mathcal{T}$ of sets of states by

$$
\mathcal{T}=\{P \mid \exists q \in F \text { such that } P \rightarrow q \in E\} .
$$

The path $\gamma$ is then successful if and only if $q_{0}$ is initial and the set $\lim _{\omega^{-}} \gamma$ of states belongs to the family $\mathcal{T}$. This matches the definition of a successful path in a Muller automaton [24, p. 148].

The set of cuts of an ordinal $\alpha$ is the ordinal $\alpha+1$. Therefore, the notion of path we have introduced coincides for ordinal words with the notion of path considered in [2].

$$
\left|\begin{array}{llllllllllllllll}
0 & 1 & 1 & 1 & 2 & 2 & 2 & 0 & 1 & 1 & 1 & 2 & 2 & 2 & 0 & 0
\end{array}\right|
$$

Figure 3: A path labeled by $\left(b^{-\omega} a b^{\omega}\right)^{2}$

Example 18 Consider the automaton $\mathcal{A}$ of Figure 2 and let $x$ be the word $\left(b^{-\omega} a b^{\omega}\right)^{2}$ of length $\mathbb{Z}+\mathbb{Z}$. A successful path $\gamma$ labeled by $x$ is pictured in Figure 3 . This path is made of two copies of the path $01^{-\omega} 2^{\omega} 0$. A path for the word $\left(b^{-\omega} a b^{\omega}\right)^{\zeta}$ cannot be made by $\mathbb{Z}$ copies of $01^{-\omega} 2^{\omega} 0$ because the right limit set of the first state would be $\{0,1,2\}$ and the automaton has no transition of the form $q \rightarrow\{0,1,2\}$. The automaton $\mathcal{A}$ recognizes the set $\left(b^{-\omega} a b^{\omega}\right)^{*}$.

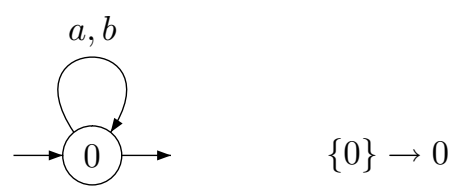

Figure 4: Automaton of Example 19

Example 19 Consider the automaton $\mathcal{A}$ pictured in Figure 4. This automaton has no right limit transition. It recognizes the words whose length is an ordinal since a linear ordering $J$ is an ordinal if and only if each of its cuts except the last has a successor in $\hat{J}$. The automaton obtained by suppressing the left limit transition of $\mathcal{A}$ recognizes the set of finite words since a linear ordering $J$ is finite if and only if each of its cuts except the first has a predecessor in $\hat{J}$ and each of its cuts except the last a successor in $\hat{J}$.

\section{Rational expressions vs automata}

In this section, we prove that rational expressions and automata are equivalent. This means that a set of words on linear orderings is rational if and only if it can 
be recognized by an automaton. This result extends Kleene's well-known theorem for finite words. Kleene's theorem was first extended to words of length $\omega$ by Büchi $[7]$ and was later extended to words of ordinal length by Wojciechowski [25]. We have then the following theorem, which is the main result.

Theorem 20 A set of words on countable scattered linear orderings is rational if and only if it is recognizable.

The following two examples illustrate the theorem.

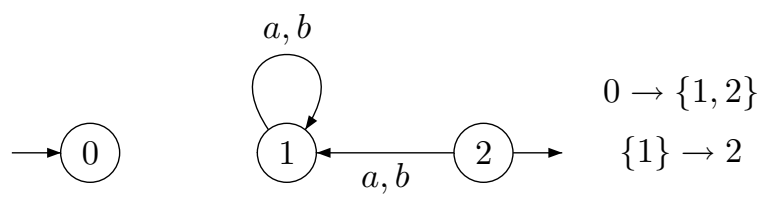

Figure 5: Automaton recognizing $\left(A^{\omega}\right)^{-\omega}$

Example 21 Let $A$ be the alphabet $\{a, b\}$. The automaton pictured in Figure 5 recognizes the set denoted by the rational expression $\left(A^{\omega}\right)^{-\omega}$. The part of the automaton given by state 1 and the left limit transition $\{1\} \rightarrow 2$ recognizes the set $A^{\omega}$. The successor transition from state 2 to state 1 allows the concatenation of two words of $A^{\omega}$. The right limit transition $0 \rightarrow\{1,2\}$ leads to a sequence of length $-\omega$ of words of $A^{\omega}$.

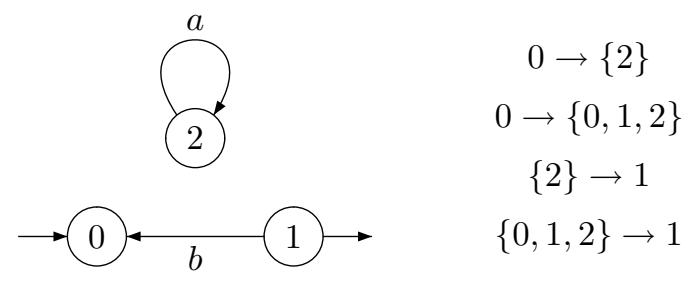

Figure 6: Automaton recognizing $a^{\zeta} \diamond b$

Example 22 The automaton pictured in Figure 6 recognizes the set denoted by the rational expression $a^{\zeta} \diamond b$. The part of the automaton given by state 2 and the two limit transitions $0 \rightarrow\{2\}$ and $\{2\} \rightarrow 1$ accepts the word $a^{\zeta}$ whereas the part given by the successor transition from state 1 to state 0 accepts the word $b$. Each occurrence of $a^{\zeta}$ (except the first) is preceded by an occurrence of $b$ in the automaton, and each occurrence of $a^{\zeta}$ (except the last) is followed by an occurrence of $b$. More generally, thanks to the limit transitions $0 \rightarrow\{0,1,2\}$ and $\{0,1,2\} \rightarrow 1$, the occurrences of $a^{\zeta}$ are indexed by a linear ordering $J \in \mathcal{S} \backslash\{\varnothing\}$, 
the occurrences of $b$ are indexed by the ordering $\hat{J}^{*}$ and they are interleaved according to the ordering $J \cup \hat{J}^{*}$.

The next two sections are devoted to the proof of the theorem. The first section contains the proof that a rational set is recognizable and the second section contains the proof of the converse.

\subsection{From rational expressions to automata}

In this section, we prove that any rational set of words is recognized by an automaton. The proof is by induction on the structure of the rational expression denoting the set. For each rational operation, we describe a corresponding construction for the automata. The constructions for union, concatenation, and finite iteration are very similar to the classical ones for automata on finite words [18, p. 15].

In order to combine two automata, it is convenient if no transition enters an initial state or leaves a final state. Furthermore, some problems may come from the empty word. To overcome them, any set $X$ may be decomposed as $X=X^{\prime}+\delta(X)$, where $X^{\prime}$ is $X-\{\varepsilon\}$ and $\delta(X)$ is $\{\varepsilon\}$ if $\varepsilon \in X$ or $\varnothing$ otherwise.

An automaton is said to be normalized if it has a unique initial state $i$ and a unique final state $f \neq i$ and it has no transition which enters $i$ or leaves $f$. Note that these conditions imply that the states $i$ and $f$ can only occur as the first state and the last state of a path. Therefore transitions of the form $P \rightarrow q$ or $q \rightarrow P$ where $P$ contains $i$ or $f$ cannot occur in a path. In the sequel, we assume that a normalized automaton does not have transitions of the form $P \rightarrow q$ or $q \rightarrow P$ where $P$ contains $i$ or $f$.

The following lemma states that the empty word can be added or removed without changing recognizability, and that a recognizable set which does not contain the empty word can be recognized by a normalized automaton. Note that this condition is necessary since a normalized automaton cannot accept the empty word.

Lemma 23 Let $X$ be a set of words. The set $X$ is recognizable if and only if $X+\varepsilon$ is recognizable. Furthermore if $\varepsilon \notin X$, then $X$ can be recognized by a normalized automaton.

Proof Suppose that $X$ is recognized by the automaton $\mathcal{A}=(Q, E, I, F)$. The set $X+\varepsilon$ is recognized by the automaton $\mathcal{A}^{\prime}$ obtained by adding a new initial and final state $i$. Let $Q^{\prime}$ be the set $Q \cup\{i\}$ where $i$ does not belong to $Q$. The automaton $\mathcal{A}^{\prime}$ is equal to $\left(Q^{\prime}, E^{\prime}, I^{\prime}, F^{\prime}\right)$ where $E^{\prime}=E, I^{\prime}=I \cup\{i\}$ and $F^{\prime}=F \cup\{i\}$.

Conversely, suppose that the set $X+\varepsilon$ is recognized by the automaton $\mathcal{A}=(Q, E, I, F)$. Without loss of generality, we may assume $\varepsilon \notin X$. The set $X$ is then recognized by the normalized automaton $\mathcal{A}^{\prime}$ obtained by modifying $\mathcal{A}$ as follows. Let $Q^{\prime}$ be the set $Q \cup\{i, f\}$ where $i$ and $f$ are new states that do 
not belong to $Q$. Define the set $E^{\prime}$ of transitions by

$$
\begin{aligned}
E^{\prime}=E & \cup\{i \stackrel{a}{\rightarrow} f \mid \exists p \stackrel{a}{\rightarrow} q \text { with } p \in I \text { and } q \in F\} \\
& \cup\{i \stackrel{a}{\rightarrow} q \mid \exists p \stackrel{a}{\rightarrow} q \text { with } p \in I\} \cup\{i \rightarrow P \mid \exists p \rightarrow P \text { with } p \in I\} \\
& \cup\{p \stackrel{a}{\rightarrow} f \mid \exists p \stackrel{a}{\rightarrow} q \text { with } q \in F\} \cup\{P \rightarrow f \mid \exists P \rightarrow q \text { with } q \in F\} .
\end{aligned}
$$

It is then straightforward to check that the automaton $\mathcal{A}^{\prime}=\left(Q^{\prime}, E^{\prime},\{i\},\{f\}\right)$ recognizes $X$. Indeed, a non-empty word $x$ labels a successful path $\gamma$ in $\mathcal{A}$ if and only if it labels the successful path $\gamma^{\prime}$ in $\mathcal{A}^{\prime}$ obtained by replacing the first and last states of $\gamma$ by $i$ and $f$.

We claim that if both sets $X_{1}$ and $X_{2}$ are recognizable, then the sets $X_{1}+$ $X_{2}, X_{1} X_{2}, X_{1}^{*}, X_{1}^{\omega}, X_{1}^{-\omega}, X_{1}^{\sharp}, X_{1}^{-\sharp}$ and $X_{1} \diamond X_{2}$ are also recognizable. For each of these rational operations, we describe a corresponding construction on automata.

Suppose that the sets $X_{1}$ and $X_{2}$ are recognized by the automata $\mathcal{A}_{1}=$ $\left(Q_{1}, E_{1}, I_{1}, F_{1}\right)$ and $\mathcal{A}_{2}=\left(Q_{2}, E_{2}, I_{2}, F_{2}\right)$. Without loss of generality, we may assume that $Q_{1}$ and $Q_{2}$ are disjoint. The set $X_{1}+X_{2}$ is then recognized by the automaton $\mathcal{A}$ obtained by mere juxtaposition of the two automata $\mathcal{A}_{1}$ and $\mathcal{A}_{2}$. This automaton $\mathcal{A}$ is equal to $\mathcal{A}=(Q, E, I, F)$ where $Q=Q_{1} \cup Q_{2}, E=E_{1} \cup E_{2}$, $I=I_{1} \cup I_{2}$ and $F=F_{1} \cup F_{2}$. Note that this construction does not require that the automata recognizing $X_{1}$ and $X_{2}$ be normalized.

In order to define formally the constructions for the other rational operations, we introduce the following notation. Let $\mathcal{A}$ be an automaton $(Q, E, I, F)$ and let $q$ be one of its states. We denote by $E[p \leftarrow q]$ the set of transitions obtained by replacing by $p$ each occurrence of $q$ in each transition of $E$.

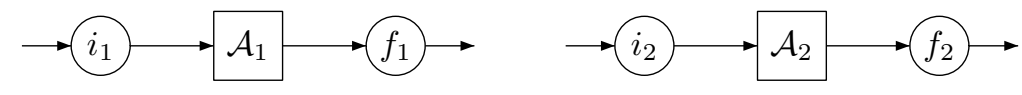

Figure 7: Automata $\mathcal{A}_{1}$ and $\mathcal{A}_{2}$

The constructions on automata corresponding to the other rational operations require that the automata be normalized. Define $X_{1}^{\prime}$ and $X_{2}^{\prime}$ by $X_{1}^{\prime}=$ $X_{1}-\varepsilon$ and $X_{2}^{\prime}=X_{2}-\varepsilon$. The sets $X_{1}$ and $X_{2}$ are equal to $X_{1}^{\prime}+\delta\left(X_{1}\right)$ and $X_{2}^{\prime}+\delta\left(X_{2}\right)$. By Lemma 23, the sets $X_{1}^{\prime}$ and $X_{2}^{\prime}$ are recognized by two normalized automata $\mathcal{A}_{1}=\left(Q_{1}, E_{1},\left\{i_{1}\right\},\left\{f_{1}\right\}\right)$ and $\mathcal{A}_{2}=\left(Q_{2}, E_{2},\left\{i_{2}\right\},\left\{f_{2}\right\}\right)$ pictured in Figure 7. In the figure, all states which are neither initial nor final are represented by a squared box. Without loss of generality, we may assume that $Q_{1}$ and $Q_{2}$ are disjoint.

The set $X_{1} X_{2}$ is equal to $X_{1}^{\prime} X_{2}^{\prime}+\delta\left(X_{1}\right) X_{2}^{\prime}+X_{1}^{\prime} \delta\left(X_{2}\right)+\delta\left(X_{1}\right) \delta\left(X_{2}\right)$. Since the class of recognizable sets is closed under union, it suffices to construct an automaton for $X_{1}^{\prime} X_{2}^{\prime}$. This set is recognized by the automaton $\mathcal{A}$ obtained by first juxtaposing the automata $\mathcal{A}_{1}$ and $\mathcal{A}_{2}$ and then merging the final state $f_{1}$ of $\mathcal{A}_{1}$ and the initial state $i_{2}$ of $\mathcal{A}_{2}$ into a state called $f_{1}$ which is neither initial 


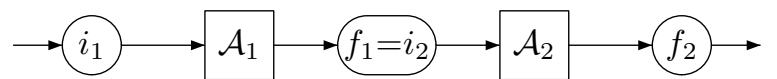

Figure 8: Automaton for $X_{1}^{\prime} X_{2}^{\prime}$

nor final. This construction is pictured in Figure 8. The automaton $\mathcal{A}$ is equal to $\left(Q, E,\left\{i_{1}\right\},\left\{f_{2}\right\}\right)$ where $Q=Q_{1} \cup Q_{2}-\left\{i_{2}\right\}$ and $E=E_{1} \cup E_{2}\left[f_{1} \leftarrow i_{2}\right]$. Suppose that the words $x_{1}$ and $x_{2}$ label the successful paths $\gamma_{1}$ and $\gamma_{2}$ in $\mathcal{A}_{1}$ and $\mathcal{A}_{2}$. Since $i_{2}$ is identified with $f_{1}$, the two paths $\gamma_{1}$ and $\gamma_{2}$ can be concatenated to form a successful path in $\mathcal{A}$ labeled by $x_{1} x_{2}$. Conversely, the state $f_{1}=i_{2}$ occurs at least once in any successful path in $\mathcal{A}$ and it occurs exactly once since no transition leaves $f_{1}$ in $\mathcal{A}_{1}$ and no transition enters $i_{2}$ in $\mathcal{A}_{2}$. Therefore, any successful path in $\mathcal{A}$ is the concatenation of successful paths in $\mathcal{A}_{1}$ and $\mathcal{A}_{2}$. This proves that $\mathcal{A}$ recognizes the set $X_{1}^{\prime} X_{2}^{\prime}$.

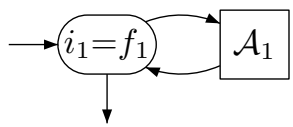

Figure 9: Automaton for $X_{1}^{*}$

The set $X_{1}^{*}=X_{1}^{\prime *}$ is recognized by the automaton $\mathcal{A}$ obtained by merging the initial state $i_{1}$ and final state $f_{1}$ of $\mathcal{A}_{1}$ into a state which is both initial and final. This construction is pictured in Figure 9. More formally, the automaton $\mathcal{A}$ is equal to $\left(Q, E,\left\{i_{1}\right\},\left\{i_{1}\right\}\right)$ where $Q=Q_{1}-\left\{f_{1}\right\}$ and $E=E_{1}\left[i_{1} \leftarrow f_{1}\right]$. Suppose that $x_{1}, \ldots, x_{n}$ are accepted by $\mathcal{A}_{1}$. The successful paths $\gamma_{1}, \ldots, \gamma_{n}$ in $\mathcal{A}_{1}$ labeled by $x_{1}, \ldots, x_{n}$ can be concatenated to form a successful path in $\mathcal{A}$. Conversely, there are finitely many occurrences of the state $i_{1}=f_{1}$ in a successful path in $\mathcal{A}$ since there is no limit transition $P \rightarrow q$ or $q \rightarrow P$ with $P$ containing $i_{1}$. Therefore, a successful path in $\mathcal{A}$ is the concatenation of a finite number of successful paths in $\mathcal{A}_{1}$. This proves that $\mathcal{A}$ recognizes the set $X_{1}^{*}=X_{1}^{\prime *}$.

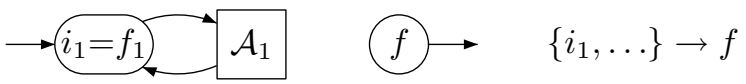

Figure 10: Automaton for $X_{1}^{\prime \omega}$

The set $X_{1}^{\omega}$ is equal to $\delta\left(X_{1}\right) X_{1}^{\prime *}+X_{1}^{\prime \omega}$. Since we have already proved that the class of recognizable sets is closed union and finite iteration, it suffices to construct an automaton for set $X_{1}^{\prime \omega}$. This set is recognized by the automaton $\mathcal{A}$ obtained by first merging the initial state $i_{1}$ and final state $f_{1}$ of $\mathcal{A}_{1}$ into a state called $i_{1}$ which is initial but not final, and then by adding a new final state $f$ 
together with all left limit transitions $P \rightarrow f$ where $P$ contains $i_{1}$. The construction is pictured in Figure 10. The automaton $\mathcal{A}$ is equal to $\left(Q, E,\left\{i_{1}\right\},\{f\}\right)$ where $Q=Q_{1} \cup\{f\}-\left\{f_{1}\right\}$ and $E=E_{1}\left[i_{1} \leftarrow f_{1}\right] \cup\left\{P \rightarrow f \mid i_{1} \in P\right\}$. Suppose that the words $x_{0}, x_{1}, x_{2}, \ldots$ are accepted by $\mathcal{A}_{1}$. The successful paths $\gamma_{0}, \gamma_{1}, \gamma_{2}, \ldots$ in $\mathcal{A}_{1}$ labeled by $x_{0}, x_{1}, x_{2}, \ldots$ can be concatenated with an additional state $f$ at the end to form a successful path in $\mathcal{A}$. Conversely, there are exactly $\omega$ occurrences of the state $i_{1}=f_{1}$ in a successful path in $\mathcal{A}$ since the only transitions entering the final state are the left limit transitions $P \rightarrow f$ with $i_{1} \in P$. Furthermore, the least upper bound of these occurrences is the last cut since there is no left limit transition $P \rightarrow q$ with $i_{1} \in P$ and $q \neq f$. Therefore, a successful path in $\mathcal{A}$ is the concatenation of $\omega$ successful paths in $\mathcal{A}_{1}$. This proves that $\mathcal{A}$ recognizes the set $X_{1}^{\prime \omega}$.

An automaton recognizing $X_{1}^{-\omega}$ is similar to the automaton for $X^{\omega}$.

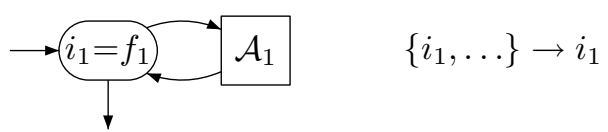

Figure 11: Automaton for $X_{1}^{\sharp}$

The set $X_{1}^{\sharp}=X_{1}^{\prime \sharp}$ is recognized by the automaton $\mathcal{A}$ obtained by first merging the initial state $i_{1}$ and the final state $f_{1}$ into a state called $i_{1}$ which is both initial and final, and then by adding all left limit transitions $P \rightarrow i_{1}$ where $P$ contains $i_{1}$. The construction is pictured in Figure 11. The automaton $\mathcal{A}$ is equal to $\left(Q, E,\left\{i_{1}\right\},\left\{i_{1}\right\}\right)$ where $Q=Q_{1}-\left\{f_{1}\right\}$ and $E=E_{1}\left[i_{1} \leftarrow f_{1}\right] \cup\{P \rightarrow$ $\left.i_{1} \mid i_{1} \in P\right\}$. Let $\alpha$ be a countable ordinal and suppose that each word $x_{\beta}$ is accepted by $\mathcal{A}_{1}$ for $\beta<\alpha$. Let $\gamma_{\beta}$ be a successful path labeled by $x_{\beta}$. Suppose first that $\alpha$ is not a limit ordinal. Thus the sequence $\gamma$ of states obtained by concatenating the paths $\gamma_{\beta}$ is a successful path in $\mathcal{A}$ since this automaton has the transitions $P \rightarrow i_{1}$ where $i_{1} \in P$. In case $\alpha$ is a limit ordinal, the paths $\gamma_{\beta}$ are concatenated with the additional state $i_{1}$ at the end to form a successful path in $\mathcal{A}$. Conversely, since the automaton $\mathcal{A}$ does not have any transition $q \rightarrow P$ where $i_{1} \in P$, the ordering of the occurrences of the state $i_{1}$ in a successful path is well-ordered. Therefore, a successful path in $\mathcal{A}$ is the concatenation of a sequence of successful paths in $\mathcal{A}_{1}$ indexed by some countable ordinal. This proves that $\mathcal{A}$ recognizes the set $X_{1}{ }^{\sharp}$.

An automaton recognizing $X_{1}^{-\sharp}$ is similar to the automaton for $X_{1}^{\sharp}$.

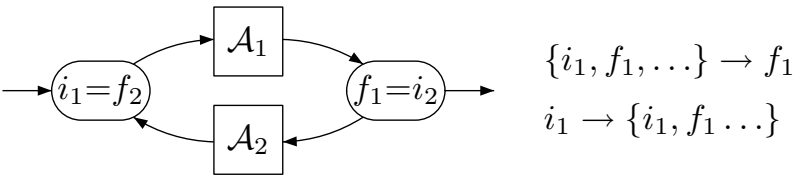

Figure 12: Automaton for $X_{1} \diamond X_{2}$ 
For the set $X_{1} \diamond X_{2}$, we first suppose that $X_{1}$ and $X_{2}$ do not contain the empty word. They are then recognized by the normalized automata $\mathcal{A}_{1}$ and $\mathcal{A}_{2}$. The set $X_{1} \diamond X_{2}$ is recognized by the automaton $\mathcal{A}$ obtained by juxtaposing the automata $\mathcal{A}_{1}$ and $\mathcal{A}_{2}$, by merging the initial state $i_{1}$ of $\mathcal{A}_{1}$ and the final state $f_{2}$ of $\mathcal{A}_{2}$ into an initial state called $i_{1}$, by merging the initial state $i_{2}$ of $\mathcal{A}_{2}$ and the final state $f_{1}$ of $\mathcal{A}_{1}$ into a final state called $f_{1}$, and by adding all the left limit transitions $P \rightarrow f_{1}$ and all the right limit transitions $i_{1} \rightarrow P$ where $P$ contains both $i_{1}$ and $f_{1}$. The construction is pictured in Figure 12 . The automaton $\mathcal{A}$ is then equal to $\left(Q, E,\left\{i_{1}\right\},\left\{f_{1}\right\}\right)$ where $Q=Q_{1} \cup Q_{2}-\left\{i_{2}, f_{2}\right\}$, $E=E_{1} \cup E_{2}\left[i_{1} \leftarrow f_{2}\right]\left[f_{1} \leftarrow i_{2}\right] \cup\left\{P \rightarrow f_{1} \mid i_{1}, f_{1} \in P\right\} \cup\left\{i_{1} \rightarrow P \mid i_{1}, f_{1} \in P\right\}$. Let $J$ be a non-empty countable scattered linear ordering. If the words $\left(x_{j}\right)_{j \in J}$ are accepted by $\mathcal{A}_{1}$ and if the words $\left(y_{c}\right)_{c \in \hat{J}^{*}}$ are accepted by $\mathcal{A}_{2}$, the successful paths labeled by these words can be interleaved and concatenated to form a path in $\mathcal{A}$ since this automaton has all the appropriate limit transitions. Conversely, a successful path in $\mathcal{A}$ is made of intervals which either contain states of $\mathcal{A}_{1}$ or states of $\mathcal{A}_{2}$. Applying Lemma 8 to the ordering $K$ of these intervals, a path in $\mathcal{A}$ can be decomposed as the concatenation of successful paths $\left(\gamma_{j}\right)_{j \in J}$ in $\mathcal{A}_{1}$ and successful paths $\left(\gamma_{c}\right)_{c \in \hat{J}^{*}}$ in $\mathcal{A}_{2}$. This proves that $\mathcal{A}$ recognizes the set $X_{1} \diamond X_{2}$.

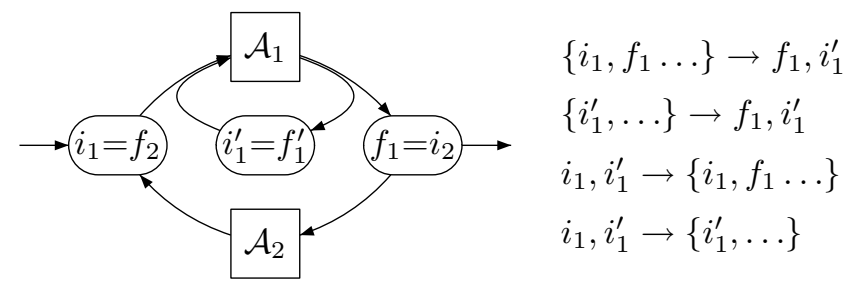

Figure 13: Automaton for $X_{1} \diamond\left(X_{2}+\varepsilon\right)$

If either $X_{1}$ or $X_{2}$ contains the empty word, the previous construction must be slightly adapted but it remains essentially the same. We describe it for the case where $X_{2}$ contains the empty word but $X_{1}$ does not. The other cases are very similar. The construction is as follows. The initial state $i_{1}$ of $\mathcal{A}_{1}$ is duplicated. This means that a new initial state $i_{1}^{\prime}$ is added, and new transitions $i_{1}^{\prime} \stackrel{a}{\rightarrow} q$ and $i_{1}^{\prime} \rightarrow P$ are added whenever $i_{1} \stackrel{a}{\rightarrow} q$ and $i_{1} \rightarrow P$ are transitions of $\mathcal{A}_{1}$. Analogously, the final state $f_{1}$ is duplicated by adding a new final state $f_{1}^{\prime}$. The automaton $\mathcal{A}$ recognizing $X_{1} \diamond X_{2}$ is obtained by juxtaposing the automata $\mathcal{A}_{1}$ and $\mathcal{A}_{2}$, merging the two states $i_{1}^{\prime}$ and $f_{1}^{\prime}$, merging the initial state $i_{1}$ of $\mathcal{A}_{1}$ and the final state $f_{2}$ of $\mathcal{A}_{2}$ into an initial state called $i_{1}$, merging the initial state $i_{2}$ of $\mathcal{A}_{2}$ and the final state $f_{1}$ of $\mathcal{A}_{1}$ into a final state called $f_{1}$, and adding all the left limit transitions $P \rightarrow f_{1}$ and $P \rightarrow i_{1}^{\prime}$ and all right limit transitions $i_{1} \rightarrow P$ and $i_{1}^{\prime} \rightarrow P$ where $P$ either contains $i_{1}^{\prime}$ or contains both $i_{1}$ and $f_{1}$. The construction is pictured in Figure 13.

We have now shown that for each rational operation, there is a corresponding construction on automata. For the base cases of the induction, we note that $\varnothing$ 
is recognized by the automaton $(\{i, f\}, \varnothing,\{i\},\{f\})$, and for each letter $a$, the set $\{a\}$ is recognized by the automaton $(\{i, f\},\{i \stackrel{a}{\rightarrow} f\},\{i\},\{f\})$. Thus, for each rational expression, there is an automaton recognizing the set denoted by that expression.

\subsection{From automata to rational expressions}

In this section, we prove that for any automaton $\mathcal{A}$, there is a rational expression denoting the set of words recognized by $\mathcal{A}$. We first introduce some notation. Let $\mathcal{A}=(Q, E, I, F)$ be a fixed automaton. The content $\mathrm{C}(\gamma)$ of a path $\gamma$ is the set of states which occur inside $\gamma$. It does not take into account the first and the last state of the path. Recall that a path $\gamma$ labeled by a word of length $J$ is a function from $\hat{J}$ into $Q$. The content of a path $\gamma$ is thus formally defined by $\mathrm{C}(\gamma)=\gamma\left(\hat{J}^{*}\right)$. Recall that $\operatorname{Out}(P)$ and $\operatorname{In}(P)$ respectively denote the set of transitions that leave and enter a state in $P$.

A path $\gamma$ from state $p$ to state $p^{\prime}$ having content $P$ and labeled by $x$ is denoted by

$$
p \underset{P}{\stackrel{x}{\longrightarrow}} p^{\prime}
$$

If $x \neq \varepsilon$, the path $\gamma$ uses a first transition $\sigma$ which leaves $p$ and a last transition $\sigma^{\prime}$ which enters $p^{\prime}$. To emphasize the use of $\sigma$ and $\sigma^{\prime}$, the path $\gamma$ is then denoted

$$
\sigma \underset{P}{\stackrel{x}{\longrightarrow}} \sigma^{\prime}
$$

In both notations, we may omit the label or the content of the path if they are not relevant.

In the proof, we often decompose a path into several paths. Given a path $\gamma$ of length $\hat{J}$ and two cuts $c<c^{\prime}$ of $J$, the path denoted by $\gamma\left[c, c^{\prime}\right]$ is the part of $\gamma$ from $c$ to $c^{\prime}$.

Let $P$ be a subset of $Q$ and let $\sigma$ and $\sigma^{\prime}$ be two transitions of $\mathcal{A}$. We define the sets of words $\prod_{\sigma, \sigma^{\prime}}^{P}, \nabla_{\sigma, \sigma^{\prime}}^{P}, \Delta_{\sigma, \sigma^{\prime}}^{P}$, and $\Gamma_{\sigma, \sigma^{\prime}}^{P}$ as follows.

$$
\begin{aligned}
& \Pi_{\sigma, \sigma^{\prime}}^{P}=\left\{x \mid \exists \gamma \text { of the form } \sigma \underset{P}{\stackrel{x}{\leftrightarrow}} \sigma^{\prime}\right\} \\
& \nabla_{\sigma, \sigma^{\prime}}^{P}=\left\{x \mid \exists \gamma \text { of the form } \sigma \underset{P}{\stackrel{x}{\leftrightarrow}} \sigma^{\prime} \text { without any transition } P \rightarrow r\right\} \\
& \Delta_{\sigma, \sigma^{\prime}}^{P}=\left\{x \mid \exists \gamma \text { of the form } \sigma \underset{P}{\stackrel{x}{\leftrightarrow}} \sigma^{\prime} \text { without any transition } r \rightarrow P\right\} \\
& \Gamma_{\sigma, \sigma^{\prime}}^{P}=\left\{x \mid \exists \gamma \text { of the form } \sigma \underset{P}{\stackrel{x}{x}} \sigma^{\prime} \text { without any transition } r \rightarrow P \text { or } P \rightarrow r\right\} .
\end{aligned}
$$

Note that without any transition $P \rightarrow r$ means that the path $\gamma$ does not use any left limit transition of the form $P \rightarrow r$ for any $r \in Q$ except perhaps for the last transition if $\sigma^{\prime}$ is a left limit transition of this form. Thus, the left limit $\lim _{c^{-}} \gamma$ at any cut $c$ different from the last cut must be a proper subset of $P$. This fact will be used in the arguments that follow. 
Both $\nabla_{\sigma, \sigma^{\prime}}^{P}$ and $\Delta_{\sigma, \sigma^{\prime}}^{P}$ are subsets of $\Pi_{\sigma, \sigma^{\prime}}^{P}$ and the set $\Gamma_{\sigma, \sigma^{\prime}}^{P}$ is equal to the intersection $\nabla_{\sigma, \sigma^{\prime}}^{P} \cap \Delta_{\sigma, \sigma^{\prime}}^{P}$.

The paths considered in the definition of the sets $\Pi_{\sigma, \sigma^{\prime}}^{P}, \nabla_{\sigma, \sigma^{\prime}}^{P}, \Delta_{\sigma, \sigma^{\prime}}^{P}$, and $\Gamma_{\sigma, \sigma^{\prime}}^{P}$ use at least one transition. Therefore, the empty word is not contained in them. Since a path is successful if its first and last states are respectively initial and final, the set of words recognized by the automaton $\mathcal{A}$ is equal to the union

$$
\delta(\mathcal{A})+\underset{P \subseteq Q, \sigma \in \operatorname{Out}(I), \sigma^{\prime} \in \operatorname{In}(F)}{\bigcup_{\sigma, \sigma^{\prime}}^{P}}
$$

where $\delta(\mathcal{A})$ is equal to $\varepsilon$ if $I \cap F \neq \varnothing$ and to $\varnothing$ otherwise. We claim that every set of the form $\Pi_{\sigma, \sigma^{\prime}}^{P}, \nabla_{\sigma, \sigma^{\prime}}^{P}, \Delta_{\sigma, \sigma^{\prime}}^{P}$, or $\Gamma_{\sigma, \sigma^{\prime}}^{P}$ is rational. The proof is by induction on the cardinality of $P$.

We first suppose that $P$ is the empty set $\varnothing$. If both transitions $\sigma$ and $\sigma^{\prime}$ are equal to the same successor transition $p \stackrel{a}{\rightarrow} q$, all four sets $\Pi_{\sigma, \sigma^{\prime}}^{P}, \nabla_{\sigma, \sigma^{\prime}}^{P}, \Delta_{\sigma, \sigma^{\prime}}^{P}$ and $\Gamma_{\sigma, \sigma^{\prime}}^{P}$ are equal to the singleton $\{a\}$. Otherwise, they are all empty. In both cases, they are rational. This completes the base case of the induction.

The rest of the proof is devoted to the induction step. We suppose that $P$ is non-empty and that for any $R \subsetneq P$ and any transitions $\tau$ and $\tau^{\prime}$, all four sets $\Pi_{\tau, \tau^{\prime}}^{R}, \nabla_{\tau, \tau^{\prime}}^{R}, \Delta_{\tau, \tau^{\prime}}^{R}$ and $\Gamma_{\tau, \tau^{\prime}}^{R}$ are rational. We first prove in Section 6.2.1 that the set $\Gamma_{\sigma, \sigma^{\prime}}^{P}$ has a rational expression over the sets $\Pi_{\tau, \tau^{\prime}}^{R}$ for $R \subsetneq P$. By the induction hypothesis and by Lemma 14 , the set $\Gamma_{\sigma, \sigma^{\prime}}^{P}$ is rational. Then we prove in Section 6.2.2 that both sets $\nabla_{\sigma, \sigma^{\prime}}^{P}$ and $\Delta_{\sigma, \sigma^{\prime}}^{P}$ have rational expressions over the sets $\Gamma_{\tau, \tau^{\prime}}^{P}$ studied in Section 6.2 .1 and the sets $\Pi_{\tau, \tau^{\prime}}^{R}, \nabla_{\tau, \tau^{\prime}}^{R}, \Delta_{\tau, \tau^{\prime}}^{R}$ and $\Gamma_{\tau, \tau^{\prime}}^{R}$ for $R \subsetneq P$. By the induction hypothesis and by Lemma $13, \nabla_{\sigma, \sigma^{\prime}}^{P}$ and $\Delta_{\sigma, \sigma^{\prime}}^{P}$ are rational. Finally, we prove in Section 6.2 .3 that the set $\Pi_{\sigma, \sigma^{\prime}}^{P}$ has a rational expression over the sets $\Gamma_{\tau, \tau^{\prime}}^{P}, \nabla_{\tau, \tau^{\prime}}^{P}, \Delta_{\tau, \tau^{\prime}}^{P}$ and the sets $\Pi_{\tau, \tau^{\prime}}^{R}, \nabla_{\tau, \tau^{\prime}}^{R}, \Delta_{\tau, \tau^{\prime}}^{R}$ and $\Gamma_{\tau, \tau^{\prime}}^{R}$ for $R \subsetneq P$. Again by the induction hypothesis and by Lemma $13, \Pi_{\sigma, \sigma^{\prime}}^{P}$ is rational.

In the following section, the proof uses the Kleene theorem for usual automata accepting finite words and a Kleene-like theorem for Büchi automata accepting $\omega$-words. We refer the reader to [18] for finite words and to [19] for $\omega$-words.

\subsection{1 $\Gamma_{\sigma, \sigma^{\prime}}^{P}$ is rational}

Let $\gamma$ be a path labeled by a word $x=\left(a_{j}\right)_{j \in J}$ in $\Gamma_{\sigma, \sigma^{\prime}}^{P}$. Let $p$ and $p^{\prime}$ be the first and last states of this path. By definition of $\Gamma_{\sigma, \sigma^{\prime}}^{P}$, the path $\gamma$ does not use any limit transition $r \rightarrow P$ or $P \rightarrow r$ for any $r \in Q$, except possibly for the beginning or ending transition. We show how to decompose the path $\gamma$ in such a way as to find a rational expression for $\Gamma_{\sigma, \sigma^{\prime}}^{P}$ over the sets $\Pi_{\tau, \tau^{\prime}}^{R}$ with $R \subsetneq P$. We consider three cases depending on the form of the transitions $\sigma$ and $\sigma^{\prime}$.

- $\sigma$ is different from $p \rightarrow P$. The idea is to decompose the path $\gamma$ into a sequence of consecutive paths, each of whose content is strictly included in $P$. The way these consecutive paths are combined to give $\gamma$ will be described 
by an automaton. Kleene's theorem for finite words together with its analog for $\omega$-words will allow us to infer from the induction hypotheses that $\Gamma_{\sigma, \sigma^{\prime}}^{P}$ is rational.

Consider the cut $(K, L)$ of the ordering $\hat{J}$ defined as follows.

$$
\begin{aligned}
K & =\left\{c \in \hat{J} \mid \mathrm{C}\left(\gamma\left[c_{\min }, c\right]\right) \subsetneq P\right\} \\
L & =\left\{c \in \hat{J} \mid \mathrm{C}\left(\gamma\left[c_{\min }, c\right]\right)=P\right\}
\end{aligned}
$$

Note that $K$ is non-empty since the first cut $c_{\min }$ belongs to $K$ and that $L$ is also non-empty since the last cut $c_{\max }$ belongs to $L$. Since $\hat{J}$ is a complete ordering, $K$ has a least upper bound $c_{1}$. By definition of $(K, L)$ and of the content, this cut $c_{1}$ must belong to $K$ and it is thus equal to $\max (K)$. Note that $c_{1}$ is different from $c_{\text {min }}$ since $\sigma$ is different from $p \rightarrow P$ and that it is different from $c_{\max }$ since $L \neq \varnothing$. Therefore there are transitions $\tau_{1}^{\prime}$ and $\tau_{1}$ entering and leaving the state $q_{1}=\gamma\left(c_{1}\right)$. Let $R_{0}$ and $R^{\prime}$ be the contents $\mathrm{C}\left(\gamma\left[c_{\min }, c_{1}\right]\right)$ and $\mathrm{C}\left(\gamma\left[c_{1}, c_{\text {max }}\right]\right)$. By definition of $c_{1}$, one has $R_{0} \subsetneq P$ and $R^{\prime} \subseteq P$. If $R^{\prime} \subsetneq P$, then $x$ belongs to $\Pi_{\sigma, \tau_{1}^{\prime}}^{R_{0}} \Pi_{\tau_{1}, \sigma^{\prime}}^{R^{\prime}}$. If $R^{\prime}=P$, then $x$ belongs to $\Pi_{\sigma, \tau_{1}^{\prime}}^{R_{0}} \Gamma_{\tau_{1}, \sigma^{\prime}}^{R^{\prime}}$ and the argument given above for $\gamma$ can be repeated for the path $\gamma\left[c_{1}, c_{\max }\right]$ since $\tau_{1}$ is not equal to $q_{1} \rightarrow P$. We then get a second cut $c_{2}$ in the interval $\left(c_{1}, c_{\max }\right)$ such that $R_{1}=\mathrm{C}\left(\gamma\left[c_{1}, c_{2}\right]\right) \subsetneq P$ and $\mathrm{C}\left(\gamma\left[c_{2}, c_{\max }\right]\right) \subseteq P$.

So, we get either a finite sequence or an $\omega$-sequence of distinct cuts $c_{1}, c_{2}, \ldots$ corresponding to states $q_{1}, q_{2}, \ldots$ with related sets $\Pi_{\sigma, \tau_{1}^{\prime}}^{R_{0}}, \Pi_{\tau_{1}, \tau_{2}^{\prime}}^{R_{1}}, \ldots$, where $R_{k} \subsetneq$ $P$ and $\tau_{k}^{\prime}$ and $\tau_{k}$ are the transitions entering and leaving $q_{k}$. Note that by construction

$$
R_{k-1} \cup\left\{q_{k}\right\} \cup R_{k}=P
$$

for every positive integer $k$.

If the sequence of cuts obtained is a finite sequence $c_{1}, \ldots, c_{n}$, then the word $x \in \Gamma_{\sigma, \sigma^{\prime}}^{P}$ belongs to the finite product

$$
\Pi_{\sigma, \tau_{1}^{\prime}}^{R_{0}} \Pi_{\tau_{1}, \tau_{2}^{\prime}}^{R_{1}} \ldots \Pi_{\tau_{n-1}, \tau_{n}^{\prime}}^{R_{n-1}} \Pi_{\tau_{n}, \sigma^{\prime}}^{R_{n}}
$$

In that case, the transition $\sigma^{\prime}$ must be different from the transition $P \rightarrow p^{\prime}$ because $R_{n} \subsetneq P$. We claim that if the sequence of cuts is an $\omega$-sequence $c_{1}, c_{2}, \ldots$, then $x$ belongs to the $\omega$-product

$$
\Pi_{\sigma, \tau_{1}^{\prime}}^{R_{0}} \Pi_{\tau_{1}, \tau_{2}^{\prime}}^{R_{1}} \Pi_{\tau_{2}, \tau_{3}^{\prime}}^{R_{2}} \ldots
$$

The least upper bound $c=\sup \left\{c_{k} \mid k \geq 1\right\}$ is equal to the last cut of $J$. Indeed, Equation (3) implies that the left $\operatorname{limit} \lim _{c^{-}} \gamma$ is equal to $P$. Since the path $\gamma$ is without any left limit transition $P \rightarrow q$ for any $q \in Q$, except for the last one, $c$ is the last cut of $J$ and $\sigma^{\prime}$ must be the left limit transition $P \rightarrow p^{\prime}$.

Thus, we have proved that if $x$ belongs to $\Gamma_{\sigma, \sigma^{\prime}}^{P}$, then it belongs to a product like (4) if $\sigma^{\prime}$ is different from $P \rightarrow p^{\prime}$, or like (5) if $\sigma^{\prime}$ is equal to $P \rightarrow p^{\prime}$. We consider two cases.

We first suppose that $\sigma^{\prime}$ is different from $P \rightarrow p^{\prime}$. Let us now give an automaton $\mathcal{B}$ which describes in a precise way the possible concatenations of 
the sets $\Pi_{\tau_{k}, \tau_{k+1}^{\prime}}^{R_{k}}$ that can arise in (4). The automaton is over the alphabet $C=\left\{\Pi_{\tau, \tau^{\prime}}^{R} \mid R \subsetneq P, \tau, \tau^{\prime} \in E\right\}$. This means that each set $\Pi_{\tau, \tau^{\prime}}^{R}$ is considered as a letter.

The states of $\mathcal{B}$ are triplets $\left(R, q, R^{\prime}\right)$ where $q$ is a state of $\mathcal{A}$, and $R$ and $R^{\prime}$ are sets of states of $\mathcal{A}$ such that $R \cup\{q\} \cup R^{\prime}=P$. The automaton $\mathcal{B}$ also has initial states of the form $\left(p, R^{\prime}\right)$ for $R^{\prime} \subsetneq P$ and final states of the form $\left(R, p^{\prime}\right)$ for $R \subsetneq P$. Transitions of $\mathcal{B}$ are defined as follows. There is a transition

$$
\left(R_{1}, q_{1}, R_{1}^{\prime}\right) \stackrel{\Pi_{\tau, \tau^{\prime}}^{R}}{\longrightarrow}\left(R_{2}, q_{2}, R_{2}^{\prime}\right)
$$

if and only if $R_{1}^{\prime}=R_{2}=R, \tau$ leaves $q_{1}$ and $\tau^{\prime}$ enters $q_{2}$. There is a transition from an initial state

$$
\left(p, R_{1}^{\prime}\right) \stackrel{\Pi_{\tau, \tau^{\prime}}^{R}}{\longrightarrow}\left(R_{2}, q_{2}, R_{2}^{\prime}\right)
$$

if and only if $R_{1}^{\prime}=R_{2}=R, \tau=\sigma$ and $\tau^{\prime}$ enters $q_{2}$. Analogously, there is a transition to a final state

$$
\left(R_{1}, q_{1}, R_{1}^{\prime}\right) \stackrel{\Pi_{\tau, \tau^{\prime}}^{R}}{\longrightarrow}\left(R_{2}, p^{\prime}\right)
$$

if and only if $R_{1}^{\prime}=R_{2}=R, \tau$ leaves $q_{1}$ and $\tau^{\prime}=\sigma^{\prime}$. Note that the initial states can only occur as the first state of a path and that final states can only occur as the last state of a path. Indeed, there is no transition entering initial states and no transition leaving final states.

This automaton $\mathcal{B}$ is a usual automaton that accepts finite words over $C$. We claim that it has the following two properties.

i) If a finite sequence $\Pi_{\sigma, \tau_{1}^{\prime}}^{R_{0}} \Pi_{\tau_{1}, \tau_{2}^{\prime}}^{R_{1}} \ldots \Pi_{\tau_{n}, \sigma^{\prime}}^{R_{n}}$ as in (4) occurs as the decomposition of a word $x$ in $\Gamma_{\sigma, \sigma^{\prime}}^{P}$, it is then accepted by $\mathcal{B}$.

ii) Conversely, if a sequence $\Pi_{\sigma, \tau_{1}^{\prime}}^{R_{0}} \Pi_{\tau_{1}, \tau_{2}^{\prime}}^{R_{1}} \ldots \Pi_{\tau_{n}, \sigma^{\prime}}^{R_{n}}$ is accepted by $\mathcal{B}$, the finite product of the corresponding sets is contained in $\Gamma_{\sigma, \sigma^{\prime}}^{P}$.

Statement (i) follows directly from Equation (3). Conversely, let $w$ be a word $\Pi_{\sigma, \tau_{1}^{\prime}}^{R_{0}} \Pi_{\tau_{1}, \tau_{2}^{\prime}}^{R_{1}} \ldots \Pi_{\tau_{n}, \sigma^{\prime}}^{R_{n}}$ accepted by $\mathcal{B}$ and let $x$ be a word over $A$ belonging to the product $\Pi_{\sigma, \tau_{1}^{\prime}}^{R_{0}} \Pi_{\tau_{1}, \tau_{2}^{\prime}}^{R_{1}} \ldots \Pi_{\tau_{n}, \sigma^{\prime}}^{R_{n}}$ of the corresponding sets. The word $x$ can be factored $x=x_{0} x_{1} \ldots x_{n}$ where each $x_{i}$ belongs to $\Pi_{\tau_{i}, \tau_{i+1}^{\prime}}^{R_{i}}$. By definition of $\Pi_{\tau_{i}, \tau_{i+1}^{\prime}}^{R_{i}}$, there is in $\mathcal{A}$, a path $\tau_{i} \rightsquigarrow \tau_{i+1}$ of content $R_{i}$, labeled by $x_{i}$. By definition of the transitions of $\mathcal{B}$, these paths can be concatenated in $\mathcal{A}$ to yield a path $\sigma \rightsquigarrow \sigma^{\prime}$ labeled by $x$. By definition of the states of $\mathcal{B}$, the content of this path is $P$ but is without any transitions $r \rightarrow P$ or $P \rightarrow r$. This completes the proof of Statement (ii).

Let $W$ be the set of words accepted by $\mathcal{B}$. By Kleene's theorem, $W$ is rational. By Statements (i) and (ii), $\Gamma_{\sigma, \sigma^{\prime}}^{P}=\lambda(W)$ where the function $\lambda$ is naturally defined on $C$. By Lemma 14 and by the induction hypothesis, $\Gamma_{\sigma, \sigma^{\prime}}^{P}$ is rational. This completes the proof of the case $\sigma^{\prime}$ different from $P \rightarrow p^{\prime}$. Note that the rational expression expressing $\Gamma_{\sigma, \sigma^{\prime}}^{P}$ over the $\Pi_{\tau, \tau^{\prime}}^{R}$ only involves unions, concatenations, and finite iterations since $W$ only contains finite words. 
We now suppose that $\sigma^{\prime}$ is equal to the transition $P \rightarrow p^{\prime}$. The proof of this case is very similar, but since the products of (5) are $\omega$-products, the automaton $\mathcal{B}$ is replaced by a Büchi automaton $\mathcal{B}^{\prime}$ that accepts $\omega$-words over the alphabet $C$. We first recall that a Büchi automaton is a usual automaton with initial and final states. An infinite path in such an automaton is successful if it starts in an initial state and if it goes through a final state infinitely many times. The automaton $\mathcal{B}^{\prime}$ is almost the same as the automaton $\mathcal{B}$. The only difference is that there are no final states of the form $\left(R, p^{\prime}\right)$ and that all states of the form $\left(R, q, R^{\prime}\right)$ and $\left(p, R^{\prime}\right)$ are final. All infinite paths starting in an initial state of this Büchi automaton are thus successful.

We claim that the automaton $\mathcal{B}^{\prime}$ has the following two properties

$\left.i^{\prime}\right)$ If an $\omega$-sequence $\Pi_{\sigma, \tau_{1}^{\prime}}^{R_{0}} \Pi_{\tau_{1}, \tau_{2}^{\prime}}^{R_{1}} \Pi_{\tau_{2}, \tau_{3}^{\prime}}^{R_{2}} \ldots$ as in (5) occurs as the decomposition of a word $x$ in $\Gamma_{\sigma, \sigma^{\prime}}^{P}$, it is then accepted by $\mathcal{B}^{\prime}$.

ii') Conversely, if an $\omega$-sequence $\Pi_{\sigma, \tau_{1}^{\prime}}^{R_{0}} \Pi_{\tau_{1}, \tau_{2}^{\prime}}^{R_{1}} \Pi_{\tau_{2}, \tau_{3}^{\prime}}^{R_{2}} \ldots$ is accepted by $\mathcal{B}^{\prime}$, the $\omega$-product of the corresponding sets is contained in $\Gamma_{\sigma, \sigma^{\prime}}^{P}$

The proofs of these two statements are exactly the same as those for $\mathcal{B}$. Let $W$ be the set of $\omega$-words accepted by $\mathcal{B}^{\prime}$. By the Kleene-like theorem for Büchi automata, $W$ is rational. By Statements (i') and (ii'), $\Gamma_{\sigma, \sigma^{\prime}}^{P}=\lambda(W)$. By Lemma 14 and by the induction hypothesis, $\Gamma_{\sigma, \sigma^{\prime}}^{P}$ is rational. This completes the proof of the case $\sigma^{\prime}$ equal to $P \rightarrow p^{\prime}$. Note that the rational expression expressing $\Gamma_{\sigma, \sigma^{\prime}}^{P}$ over the $\Pi_{\tau, \tau^{\prime}}^{R}$ only involves unions, concatenations, finite iterations, and $\omega$ iterations since $W$ only contains $\omega$-words.

- $\sigma^{\prime}$ is different from $P \rightarrow p^{\prime}$. This case is symmetrical to the previous one. The $\omega$ iteration is replaced by the $-\omega$ iteration.

- $\sigma$ equals $p \rightarrow P$ and $\sigma^{\prime}$ equals $P \rightarrow p^{\prime}$. Let $c$ be a cut of $J$ such that $c_{\min }<c<c_{\max }$. The path $\gamma$ is then decomposed into two paths $\gamma_{1}=\gamma\left[c_{\min }, c\right]$ and $\gamma_{2}=\gamma\left[c, c_{\max }\right]$ and the word $x$ is factored $x=x_{1} x_{2}$ where $x_{1}$ and $x_{2}$ are the labels of $\gamma_{1}$ and $\gamma_{2}$. The content of $\gamma_{1}$ and the content of $\gamma_{2}$ are both equal to $P$ because $\sigma$ equals $p \rightarrow P$ and $\sigma^{\prime}$ equals $P \rightarrow p^{\prime}$.

Let $q$ be the state $\gamma(c)$ and let $\tau, \tau^{\prime}$ be the transitions entering and leaving $q$ at $c$. By definition of $\Gamma_{\sigma, \sigma^{\prime}}^{P}$, both transitions $\tau$ and $\tau^{\prime}$ are different from $p \rightarrow P$ and $P \rightarrow p^{\prime}$. The word $x$ belongs then to a product of the form $\Gamma_{\sigma, \tau^{\prime}}^{P} \Gamma_{\tau, \sigma^{\prime}}^{P}$ for some $\left(\tau^{\prime}, \tau\right) \in \mathcal{T}$ where

$$
\mathcal{T}=\left\{\left(\tau^{\prime}, \tau\right) \mid \exists q \quad \tau^{\prime} \in \operatorname{In}(q), \tau \in \operatorname{Out}(q) \text { and } \tau^{\prime} \neq P \rightarrow q, \tau \neq q \rightarrow P\right\} .
$$

It follows that

$$
\Gamma_{\sigma, \sigma^{\prime}}^{P} \subseteq \bigcup_{\left(\tau^{\prime}, \tau\right) \in \mathcal{T}} \Gamma_{\sigma, \tau^{\prime}}^{P} \Gamma_{\tau, \sigma^{\prime}}^{P}
$$

and since $\Gamma_{\sigma, \tau^{\prime}}^{P} \Gamma_{\tau, \sigma^{\prime}}^{P} \subset \Gamma_{\sigma, \sigma^{\prime}}^{P}$ for each $\left(\tau^{\prime}, \tau\right) \in \mathcal{T}$, the relationship is actually one of equality. The set on the right is a finite union of products of terms shown 
to be rational in the previous two cases and so is itself rational; thus $\Gamma_{\sigma, \sigma^{\prime}}^{P}$ is rational.

\subsection{2 $\Delta_{\sigma, \sigma^{\prime}}^{P}$ and $\nabla_{\sigma, \sigma^{\prime}}^{P}$ are rational}

We now prove that $\Delta_{\sigma, \sigma^{\prime}}^{P}$ is rational. The proof for $\nabla_{\sigma, \sigma^{\prime}}^{P}$ is symmetrical. Let $\gamma$ be a path labeled by a word $x=\left(a_{j}\right)_{j \in J}$ in $\Delta_{\sigma, \sigma^{\prime}}^{P}$. Let $p$ and $p^{\prime}$ be the first and the last state of this path. By definition, this path does not use any right limit transition $r \rightarrow P$, except perhaps for $\sigma$.

The idea is again to decompose the path $\gamma$ into a sequence of consecutive paths. The decomposition is performed according to the occurrences of the left limit transitions $P \rightarrow r$ in $\gamma$. The label of each path of this decomposition belongs to some set $\Gamma_{\tau, \tau^{\prime}}^{P}$ whose rationality has been proved in Section 6.2.1.

Consider the subordering $K$ of $\hat{J}$ defined by the occurrences of left limit transitions $P \rightarrow r$ in $\gamma$, that is

$$
K=\left\{c \in \hat{J} \mid \lim _{c^{-}} \gamma=P\right\} .
$$

Note that $c_{\min }$ cannot belong to $K$ because $\lim _{c_{\min }^{-}} \gamma$ is empty by definition. The last cut $c_{\max }$ belongs to $K$ if and only if $\sigma^{\prime}$ is the transition $P \rightarrow p^{\prime}$.

If $K$ is empty or contains only $c_{\max }$, then the word $x$ belongs to $\Gamma_{\sigma, \sigma^{\prime}}^{P}$. In the rest of this section, we assume that $K$ contains some cut other than $c_{\max }$. We will consider two cases depending on whether $\sigma$ is the transition $p \rightarrow P$ or not. Before considering these two cases, we state two properties of $K$ that will be useful in both cases.

We first claim that $K$ has a greatest element $\max (K)$. Let $c=\sup (K)$ be the least upper bound of $K$. The left $\operatorname{limit} \lim _{c^{-}} \gamma$ is then equal to $P$ and thus $c$ belongs to $K$. If $\sigma^{\prime}$ is equal to $P \rightarrow p^{\prime}$, then $\max (K)=c_{\max }$. Otherwise one has $\max (K)<c_{\max }$.

The ordering $K$ is not necessarily an ordinal but it is almost well-ordered. We claim that if it contains an infinite decreasing sequence $c_{0}>c_{1}>c_{2}>\cdots$, then this sequence converges to $c_{\min }$ and the transition $\sigma$ must be $p \rightarrow P$. This implies in particular that for any cut $(L, R)$ of $K$ different from $(\varnothing, K)$, the ordering $R$ is an ordinal.

Suppose that $K$ indeed contains an infinite sequence $c_{0}>c_{1}>c_{2}>\cdots$ and let $c$ be the greatest lower bound of $\left\{c_{i} \mid i \geq 0\right\}$. The right $\operatorname{limit} \lim _{c^{+}} \gamma$ is then equal to $P$. Since the path does not use any right limit transition $r \rightarrow P$, except perhaps for $\sigma$, it follows that $c$ is the first cut $c_{\min }$ and that $\sigma$ is the transition $p \rightarrow P$.

We consider two cases depending on the form of transition $\sigma$.

- $\sigma$ is different from $p \rightarrow P$. By the second property of $K$, there exists no infinite decreasing sequence in $K$, that is, $K$ is well-ordered. It follows that $K$ has a least element $\min (K)$ and that each element $c$ of $K$ different from $\max (K)$ has a successor in $K$ that we denote by $c+1$ by a slight abuse of language. Let $\tau_{c}^{\prime}$ and $\tau_{c}$ be the transitions entering and leaving the state $q_{c}=\gamma(c)$. By definition 
of $K$, the transition $\tau_{c}^{\prime}$ is equal to $P \rightarrow q_{c}$ for each $c \in K$. Let $x_{c}$ be the label of the path $\gamma[c, c+1]$. Each word $x_{c}$ belongs to $\Gamma_{\tau_{c}, \tau_{c+}^{\prime}}^{P}$. Let $y$ be the label of the path $\gamma^{\prime}=\gamma\left[c_{\min }, \min (K)\right]$. The word $y$ belongs to $\Gamma_{\sigma, \tau_{\min (K)}^{\prime}}^{\prime}$. If the transition $\sigma^{\prime}$ is different from $P \rightarrow p^{\prime}$, one has $\max (K)<c_{\max }$. In that case, we also consider the label $z$ of the path $\gamma^{\prime \prime}=\gamma\left[\max (K), c_{\max }\right]$. The word $z$ belongs to $\Gamma_{\tau_{\max (K)}, \sigma^{\prime}}^{P}$ if $\mathrm{C}\left(\gamma^{\prime \prime}\right)=P$ and it belongs to $\prod_{\tau_{\max (K)}^{R}, \sigma^{\prime}}^{R}$ if $\mathrm{C}\left(\gamma^{\prime \prime}\right)=R \subsetneq P$. If $\sigma^{\prime}$ is equal to $P \rightarrow p^{\prime}$, the word $x$ is equal to $y \prod_{c<\max (K)} x_{c}$ and if $\sigma^{\prime}$ is different from $P \rightarrow p^{\prime}$, the word $x$ is equal to $y\left(\prod_{c<\max (K)} x_{c}\right) z$.

Therefore, as $K$ is well-ordered,

$$
\Delta_{\sigma, \sigma^{\prime}}^{P} \subseteq \Gamma_{\sigma, \sigma^{\prime}}^{P} \cup\left(\bigcup_{\tau^{\prime} \in T^{\prime}} \Gamma_{\sigma, \tau^{\prime}}^{P}\right)\left(\bigcup_{\tau \in T} \Gamma_{\tau^{\prime} \in T^{\prime}}^{P} \Gamma^{\tau^{\prime}}\right)^{\sharp}
$$

if $\sigma^{\prime}$ equals $P \rightarrow p^{\prime}$, and

$$
\Delta_{\sigma, \sigma^{\prime}}^{P} \subseteq \Gamma_{\sigma, \sigma^{\prime}}^{P} \cup\left(\bigcup_{\tau^{\prime} \in T^{\prime}} \Gamma_{\sigma, \tau^{\prime}}^{P}\right)\left(\bigcup_{\tau \in T} \Gamma_{\tau^{\prime} \in T^{\prime}}^{P} \Gamma_{\tau, \tau^{\prime}}\right)^{\sharp}\left(\bigcup_{\tau \in T} \Gamma_{\tau, \sigma^{\prime}}^{P} \cup \bigcup_{\tau \in T} \Pi_{R \subsetneq P}^{R} \Pi_{\tau, \sigma^{\prime}}^{R}\right)
$$

if $\sigma^{\prime}$ is different from $P \rightarrow p^{\prime}$, where the subsets $T$ and $T^{\prime}$ of transitions are defined as follows:

$$
\begin{aligned}
T & =\{\tau \mid \exists q \in P \quad \tau \in \operatorname{Out}(q), \tau \neq q \rightarrow P \text { and } P \rightarrow q \in E\}, \\
T^{\prime} & =\{P \rightarrow q \mid q \in P \text { and } P \rightarrow q \in E\} .
\end{aligned}
$$

Let us show that inclusions (7) and (8) are in fact equalities. We first study the set

$$
X=\left(\bigcup_{\tau \in T} \Gamma_{\tau^{\prime} \in T^{\prime}}^{P} \Gamma_{\tau, \tau^{\prime}}^{\sharp} .\right.
$$

We start with a small remark concerning transitions of $T^{\prime}$. Let $\tau_{1}^{\prime}, \tau_{2}^{\prime} \in T^{\prime}$ be two transitions of the form $\tau_{1}^{\prime}=P \rightarrow q_{1}$ and $\tau_{2}^{\prime}=P \rightarrow q_{2}$. Let $\delta$ be a path ending with $\tau_{1}^{\prime}$, that is $\delta$ is a sequence of states of the form $\delta^{\prime} q_{1}$. Then $\delta^{\prime} q_{2}$ is also a path with the same label and the same content as $\delta^{\prime} q_{1}$. By this remark, we can conclude that $\Gamma_{\tau, \tau_{1}^{\prime}}^{P}=\Gamma_{\tau, \tau_{2}^{\prime}}^{P}$.

We claim that each non-empty word $x \in X$ labels a path $\delta: \tau \rightsquigarrow \tau^{\prime}$ of content $P$ for some $\tau \in T$ and $\tau^{\prime} \in T^{\prime}$, and that $\delta$ is without any right limit transition $r \rightarrow P$, for all $r \in Q$. Let $x$ be a non-empty word of $X$. It can be factored $x=\Pi_{\beta<\alpha} x_{\beta}$ where $0<\alpha<\omega_{1}$ and each word $x_{\beta}$ belongs to $\Gamma_{\tau_{\beta}, \tau_{\beta}^{\prime}}^{P}$ for some $\tau_{\beta} \in T$ and $\tau_{\beta}^{\prime} \in T^{\prime}$. Let $\delta_{\beta}$ be a path $\tau_{\beta} \rightsquigarrow \tau_{\beta}^{\prime}$ labeled by $x_{\beta}$. Define $\delta_{\beta}^{\prime}$ by $\delta_{\beta}=\delta_{\beta}^{\prime} q_{\beta}$. We claim that for any $q$ such that $P \rightarrow q$ belongs to $E$, $\delta=\left(\Pi_{\beta<\alpha} \delta_{\beta}^{\prime}\right) q$ is a path labeled by $x$. Indeed, if $\beta$ is a successor ordinal $\beta^{\prime}+1$, the first state of $\delta_{\beta}$ can replace the last state of $\delta_{\beta^{\prime}}$ due to the previous remark. If $\beta$ is a limit ordinal, the left limit of $\delta$ at the first state $q$ of $\delta_{\beta}$ is $P$ and $P \rightarrow q$ is a transition by definition of $T$. Note that the path $\delta$ is without any right limit 
transition $r \rightarrow P$, for all $r \in Q$. If $x$ is the empty word, we take for $\delta$ the empty path.

Finally a path $\delta^{\prime}$ of label $y \in \Gamma_{\sigma, \tau^{\prime}}^{P}$ with $\tau^{\prime} \in T^{\prime}$, can be concatenated with the path $\delta$ constructed above, due again to the previous remark. For the same reason, the path $\delta^{\prime} \delta$ can be concatenated with a path $\delta^{\prime \prime}$ labeled by $z$ in $\Gamma_{\tau, \sigma^{\prime}}^{P}$ or in $\Pi_{\tau, \sigma^{\prime}}^{R}$ for $R \subsetneq P$. Moreover the resulting path is without any right limit transition $r \rightarrow P$, for all $r \in Q$.

Therefore, if $\sigma^{\prime}$ equals $P \rightarrow p^{\prime}$, then equality holds in (7), otherwise equality holds in (8). This shows that $\Delta_{\sigma, \sigma^{\prime}}^{P}$ has a rational expression over the sets $\Gamma_{\tau, \tau^{\prime}}^{P}$ and the sets $\prod_{\tau, \tau^{\prime}}^{R}$ for $R \subsetneq P$. By the induction hypothesis and by Lemma 13, $\Delta_{\sigma, \sigma^{\prime}}^{P}$ is rational.

- $\sigma$ equals $p \rightarrow P$. We work again with the path $\gamma$ with label $x$ as defined at the beginning of Section 6.2.2. Two cases occur depending on the existence of an infinite decreasing sequence in the set $K$ defined by Equation (6).

We first suppose that there is no infinite decreasing sequence in $K$. The set $K$ is then well-ordered. Since $K \neq \varnothing$, it has a least element $c=\min (K)$ which is different from $c_{\text {min }}$. The path $\gamma$ can be decomposed into the two paths $\gamma_{1}=\gamma\left[c_{\min }, c\right]$ and $\gamma_{2}=\gamma\left[c, c_{\max }\right]$. Let $x_{1}, x_{2}$ be the labels of the paths $\gamma_{1}$, $\gamma_{2}$, and let $\tau^{\prime}, \tau$ be the transitions entering and leaving the state $q=\gamma(c)$. By definition of $c$, the word $x_{1}$ belongs to $\Gamma_{\sigma, \tau^{\prime}}^{P}$. The word $x_{2}$ belongs to $\Delta_{\tau, \sigma^{\prime}}^{P}$ or in $\Pi_{\tau, \sigma^{\prime}}^{R}$ with $R \subsetneq P$ according to the content of $\gamma_{2}$. Note that $\Delta_{\tau, \sigma^{\prime}}^{P}$ is such that $\tau$ is different from $q \rightarrow P$ and thus has been proved to be rational in the previous case.

This shows that

$$
x \in \bigcup_{\tau \in T} \Gamma_{\tau^{\prime} \in T^{\prime}}^{P} \Gamma_{\sigma, \tau^{\prime}}^{P}\left(\Delta_{\tau, \sigma^{\prime}}^{P} \cup \bigcup_{R \subsetneq P} \Pi_{\tau, \sigma^{\prime}}^{R}\right)
$$

where the sets $T$ and $T^{\prime}$ are defined by Equations (9) and (10).

We now suppose that there is an infinite decreasing sequence $c_{1}>c_{2}>$ $c_{3}>\cdots$ in $K$. We can assume that $c_{1}$ is different from $c_{\max }$. Otherwise $c_{1}$ is removed from the sequence to get another infinite decreasing sequence with that property. Let $q_{i}$ be the state $\gamma\left(c_{i}\right)$, and let $\tau_{i}^{\prime}$ and $\tau_{i}$ be the transitions entering and leaving the state $q_{i}$ at $c_{i}$. Let $\gamma_{i}$ be the path $\gamma\left[c_{i+1}, c_{i}\right]$. Recall that we have proved that the sequence $\left(c_{i}\right)_{i \geq 1}$ converges to $c_{\min }$. This shows that the path $\gamma\left[c_{\min }, c_{1}\right]$ can be decomposed as a sequence of length $-\omega$ of the paths $\gamma_{i}$. By definition of $\Delta_{\sigma, \sigma^{\prime}}^{P}$, each transition $\tau_{i}$ is different from the transition $q_{i} \rightarrow P$. This shows that the label $x_{i}$ of $\gamma_{i}$ belongs to $\Delta_{\tau_{i+1}, \tau_{i}^{\prime}}^{P}$ that has already been shown to be rational in the previous case. Let $x_{0}$ be label of the path $\gamma_{0}=\gamma\left[c_{1}, c_{\max }\right]$. It belongs to $\Delta_{\tau_{0}, \sigma^{\prime}}^{P}$ if $\mathrm{C}\left(\gamma_{0}\right)=P$ and it belongs to $\prod_{\tau_{0}, \sigma^{\prime}}^{R}$ if $\mathrm{C}\left(\gamma_{0}\right)=R \subsetneq P$. Thus

$$
x \in\left(\bigcup_{\tau \in T} \Delta_{\tau^{\prime} \in T^{\prime}}^{P} \Delta_{\tau, \tau^{\prime}}\right)^{-\omega}\left(\bigcup_{\tau \in T} \Delta_{\tau, \sigma^{\prime}}^{P} \cup \bigcup_{\tau \in T} \bigcup_{R \subsetneq P}^{R} \Pi_{\tau, \sigma^{\prime}}^{R}\right)
$$


where the sets $T$ and $T^{\prime}$ are defined by Equations (9) and (10).

Combining the two cases (11), (12) with the case $K=\varnothing$ or $K=\left\{c_{\max }\right\}$, one has

$$
\begin{aligned}
\Delta_{\sigma, \sigma^{\prime}}^{P} & \subseteq \Gamma_{\sigma, \sigma^{\prime}}^{P} \quad \cup \quad \bigcup_{\tau \in T, \tau^{\prime} \in T^{\prime}} \Gamma_{\sigma, \tau^{\prime}}^{P}\left(\Delta_{\tau, \sigma^{\prime}}^{P} \cup \bigcup_{R \subsetneq P} \Pi_{\tau, \sigma^{\prime}}^{R}\right) \\
& \cup\left(\bigcup_{\tau \in T} \Delta_{\tau, \tau^{\prime} \in T^{\prime}}^{P}\right)^{-\omega}\left(\bigcup_{\tau \in T} \Delta_{\tau, \sigma^{\prime}}^{P} \cup \bigcup_{\tau \in T R \subsetneq P} \Pi_{\tau, \sigma^{\prime}}^{R}\right)
\end{aligned}
$$

One can verify that this inclusion is an equality. The sets $\Delta_{\tau, \sigma^{\prime}}^{P}, \Delta_{\tau, \tau^{\prime}}^{P}$ of this equality have been shown to be rational in the previous case. By Lemma 13 and by the induction hypothesis, $\Delta_{\sigma, \sigma^{\prime}}^{P}$ is rational.

\subsection{3 $\Pi_{\sigma, \sigma^{\prime}}^{P}$ is rational}

In this last part of the proof, we show $\Pi_{\sigma, \sigma^{\prime}}^{P}$ has a rational expression over the sets $\Gamma_{\tau, \tau^{\prime}}^{P}, \nabla_{\tau, \tau^{\prime}}^{P}, \Delta_{\tau, \tau^{\prime}}^{P}$ and the sets $\Pi_{\tau, \tau^{\prime}}^{R}, \nabla_{\tau, \tau^{\prime}}^{R}, \Delta_{\tau, \tau^{\prime}}^{R}$ and $\Gamma_{\tau, \tau^{\prime}}^{R}$ for $R \subsetneq P$.

The proof is organized as follows. A path of a word in $\Pi_{\sigma, \sigma^{\prime}}^{P}$ is decomposed into small intervals using a first condensation $\sim$ (see Section 2.4 for definition). Four types of intervals are obtained by this decomposition. Then some intervals are grouped with a second condensation $\approx$ to reduce the number of types of intervals to two. Finally, Lemma 8 is used and we get a rational expression involving the binary operation $\diamond$.

Let $\gamma$ be a path labeled by a word $x=\left(a_{j}\right)_{j \in J}$ in $\Pi_{\sigma, \sigma^{\prime}}^{P}$. Let $p$ and $p^{\prime}$ be the first and the last state of this path.

Consider the condensation $\sim$ of $\hat{J}$ defined as follows. For all cuts $c_{1}, c_{2} \in \hat{J}$ where $c_{1}<c_{2}$, the relations $c_{1} \sim c_{2}$ and $c_{2} \sim c_{1}$ hold if and only if for every $c \in\left[c_{1}, c_{2}\right), \lim _{c^{+}} \gamma \neq P$ and for every $c \in\left(c_{1}, c_{2}\right], \lim _{c^{-}} \gamma \neq P$.

Any equivalence class of $\sim$ is an interval. Note that two consecutive cuts are always $\sim$-equivalent. As $\hat{J}$ is complete with a least and a greatest element, every interval can be expressed as one of the four forms $\left(c_{1}, c_{2}\right),\left(c_{1}, c_{2}\right],\left[c_{1}, c_{2}\right)$ and $\left[c_{1}, c_{2}\right]$. The form of an interval is unique because consecutive cuts are always equivalent. In the sequel we speak about the four types (), (], [) and []. Recall that an interval is right open (respectively right closed) if it has type ( ) or [) (respectively (] or []).

Let us study the structure of the equivalence classes of $\sim$ and the consecutive elements of the quotient ordering $K=\hat{J} / \sim$. Note that $K$ is a complete scattered ordering with a least and a greatest element.

Let $k \in K$ be an equivalence class of $\sim$, with $c_{1}=\inf (k)$ and $c_{2}=\sup (k)$. For any $c \in\left(c_{1}, c_{2}\right)$, one has $\lim _{c^{+}} \gamma \neq P$ and $\lim _{c^{-}} \gamma \neq P$. If $c_{1}=c_{2}$, that is, $k$ is the singleton $\left\{c_{1}\right\}$, then $\lim _{c_{1}^{-}} \gamma=\lim _{c_{1}^{+}} \gamma=P$. If $c_{1}<c_{2}$, one verifies that $k$ is left open if and only if $\lim _{c_{1}^{+}} \gamma=P$. Symmetrically, $k$ is right open if and only if $\lim _{c_{2}^{-}} \gamma=P$. 
Consider two consecutive elements $k<k^{\prime}$ of $K$. As $\hat{J}$ is complete, it is impossible that $k$ is right open and $k^{\prime}$ is left open. As consecutive cuts are equivalent modulo $\sim$, it is also impossible that $k$ is right closed and $k^{\prime}$ is left closed. Moreover, any element $k$ of $K$ which is right open always has a successor $k^{\prime}$ which is then left closed. Symmetrically, if $k$ is left open, it has a predecessor $k^{\prime}$ which is right closed.

We now define a condensation $\approx$ of the ordering $K=\hat{J} / \sim$. For all $k_{1}<$ $k_{2} \in K$, the relation

$$
k_{1} \approx k_{2}
$$

holds if and only if all elements of the interval $\left[k_{1}, k_{2}\right]$ have the same type.

As done for $K$, we study the quotient ordering $L=K / \approx$ which is a complete scattered linear ordering with a least and a greatest element.

If $l$ is an equivalence class of $\approx$ with elements of type ( ), then $l$ is a singleton. Otherwise, let $k_{1}<k_{2}$ be in $l$. As $K$ is scattered, there are two consecutive elements $k_{1}^{\prime}<k_{2}^{\prime}$ in $\left[k_{1}, k_{2}\right]$ both of type ( ). This is impossible in $K$. In the same way, any equivalence class of $\approx$ with elements of type [] is a singleton. Classes whose elements are of type (] or [), however, need not to be singletons.

Note that any equivalence class $l$ with elements of type [) always has a successor and if $l$ is left open, it also has a predecessor. Moreover, recall that an element $k$ of $K$ of the type $\left[c_{1}, c_{2}\right)$ satisfies $\lim _{c_{2}^{-}} \gamma=P$. Thus, the least upper bound $c^{\prime}=\sup \left\{c_{2} \mid\left[c_{1} c_{2}\right) \in l\right\}$ also satisfies $\lim _{c^{\prime}-\gamma}=P$. If $l$ is left open, the greatest lower bound $c=\inf \left\{c_{1} \mid\left[c_{1} c_{2}\right) \in l\right\}$ satisfies $\lim _{c^{+}} \gamma=P$. In the same way, any class $l$ with elements of type (] always has a predecessor and if $l$ is right open, it also has a successor. By similar reasoning, any class $l$ reduced to a singleton of type ( ) has a predecessor and a successor.

Consider now two consecutive elements $l<l^{\prime}$ of $L$. Since $K / \approx$ is complete, it is impossible that $l$ is right open and $l^{\prime}$ is left open. Let $k=\sup (l)$ and $k^{\prime}=\inf \left(l^{\prime}\right)$. Assume first that $l$ and $l^{\prime}$ are respectively right and left closed. Then $k$ and $k^{\prime}$ are consecutive elements of $K$, for which we already know the possible configurations. Moreover, $k$ and $k^{\prime}$ have different types since they are in different classes. Assume now that $l$ is right open and that $l^{\prime}$ is left closed. It follows that $k=k^{\prime}$ and the type of $k$ is different from the type of the elements of $l$. As $l$ is right open, two types are possible for its elements: (] or [). If the type is [), one checks that $k$ has type [] due to the properties seen for $K$. If the type of the elements of $l$ is (], then $k$ has either type [] or [). The last case when $l$ is right closed and $l^{\prime}$ is left open is symmetrical.

Let us go further. We consider $L$ as a collection of intervals of $\hat{J}$ which partition $\hat{J}$, by composing the two condensations $\sim$ and $\approx$. To avoid any confusion, when $L$ is seen as the quotient ordering over $K$, that is $L=K / \approx$, an equivalence class is described as an interval composed of elements $k$ of $K$. When $L$ is seen as the quotient ordering over $\hat{J}$, a class is described as an interval of elements $c$ of $\hat{J}$. A class $l$ is then seen as the interval $\bigcup_{k \in l} k$ of $\hat{J}$.

Let us detail the different cases. We begin by considering the case where $l$ is a singleton whose sole member $k$ has type () or []. Seen over $\hat{J}$, we respectively get $l=\left(c, c^{\prime}\right)$ or $l=\left[c, c^{\prime}\right]$. Let $\tau$ be the transition leaving $\gamma(c)$ and let $\tau^{\prime}$ be the 
transition entering $\gamma\left(c^{\prime}\right)$. Therefore, if $l=\left(c, c^{\prime}\right)$, the label $y$ of the path $\gamma\left[c, c^{\prime}\right]$ belongs to

$$
\Gamma_{\tau, \tau^{\prime}}^{P} \quad \text { with } \quad \tau=\gamma(c) \rightarrow P \text { and } \tau^{\prime}=P \rightarrow \gamma\left(c^{\prime}\right)
$$

and if $l=\left[c, c^{\prime}\right]$, then either $y=\varepsilon$ (when $c=c^{\prime}$ ) or $y$ belongs to

$$
Z_{\tau, \tau^{\prime}}=\Gamma_{\tau, \tau^{\prime}}^{P} \cup \bigcup_{R \subsetneq P} \Pi_{\tau, \tau^{\prime}}^{R} \quad \text { with } \quad \tau \neq \gamma(c) \rightarrow P \text { and } \tau^{\prime} \neq P \rightarrow \gamma\left(c^{\prime}\right) .
$$

We next consider those classes $l$ whose elements have type [). Suppose that $l$ is left open. We have seen that over $\hat{J}, l$ is an interval $l=\left(c, c^{\prime}\right)$ such that $\lim _{c^{+}} \gamma=P$ and $\lim _{c^{\prime}} \gamma=P$. The path $\gamma\left[c, c^{\prime}\right]$ is without any right limit transition $r \rightarrow P$. Let $\tau$ be the transition leaving $\gamma(c)$ and let $\tau^{\prime}$ be the transition entering $\gamma\left(c^{\prime}\right)$. Then the label $y$ of the path $\gamma\left[c, c^{\prime}\right]$ belongs to

$$
\Delta_{\tau, \tau^{\prime}}^{P} \quad \text { with } \quad \tau=\gamma(c) \rightarrow P \text { and } \tau^{\prime}=P \rightarrow \gamma\left(c^{\prime}\right) .
$$

A similar description holds for right open classes whose elements have type ( ]. With the same notation, we have $l=\left(c, c^{\prime}\right)$ and the label $y$ of the path $\gamma\left[c, c^{\prime}\right]$ belongs to

$$
\nabla_{\tau, \tau^{\prime}}^{P} \quad \text { with } \quad \tau=\gamma(c) \rightarrow P \text { and } \tau^{\prime}=P \rightarrow \gamma\left(c^{\prime}\right) .
$$

Two cases have yet to be considered: the case of left closed classes $l$ with elements of type [) and the symmetrical case of right closed classes $l$ with elements of type (]. In the former case, $l$ is an interval $l=\left[c, c^{\prime}\right)$ over $\hat{J}$ such that $\lim _{c^{+}} \gamma \neq P$ and $\lim _{c^{\prime}} \gamma=P$. The path $\gamma\left[c, c^{\prime}\right]$ is again without any right limit transition $r \rightarrow P$ and with the same notation as before, $y$ belongs to

$$
\Delta_{\tau, \tau^{\prime}}^{P} \quad \text { with } \quad \tau \neq \gamma(c) \rightarrow P \text { and } \tau^{\prime}=P \rightarrow \gamma\left(c^{\prime}\right) .
$$

In the latter case, a symmetric description holds with $l=\left(c, c^{\prime}\right]$ and $y$ belongs to

$$
\nabla_{\tau, \tau^{\prime}}^{P} \quad \text { with } \quad \tau=\gamma(c) \rightarrow P \text { and } \tau^{\prime} \neq P \rightarrow \gamma\left(c^{\prime}\right) .
$$

We now define two sets $M$ and $M^{\prime}$ such that $M \cap M^{\prime}=\varnothing$ and such that $M \cup M^{\prime}$ is a collection of intervals which partition $\hat{J}$. We define $M$ as the subordering of $L$ composed of all the elements $l$ of $L$ which are left and right open intervals $\left(c, c^{\prime}\right)$ over $\hat{J}$. These intervals are exactly those paths whose labels are described in (13), (15) and (16).

The set $M^{\prime}$ is composed of some elements of $L$ and of pairs or triples of consecutive elements of $L$ being merged into one interval. It is defined as follows. Consider an element $l=\left[c_{1}, c_{2}\right)$ of $L$ whose label is described in (17). Recall that $l$ has a successor $l^{\prime}$ which is necessarily a singleton $k$ of type []. Such a class $l^{\prime}$ has its label described in (14). The class $l^{\prime}$ is equal to an interval $l^{\prime}=\left[c_{2}, c_{3}\right]$. Analogously, if $l^{\prime \prime}=\left(c_{3}, c_{4}\right]$ is an element of $L$ whose label is described in (18), it has a predecessor $l^{\prime}=\left[c_{2}, c_{3}\right]$ whose label is described in (14). 
Let $l^{\prime}=\left[c_{2}, c_{3}\right]$ be an interval whose label is given in (14). If it has a predecessor $\left[c_{1}, c_{2}\right)$ with a label in (17) and a successor $\left(c_{3}, c_{4}\right]$ with a label in (18), then the interval $\left[c_{1}, c_{4}\right]$ obtained by fusion of the three intervals $\left[c_{1}, c_{2}\right)$, $\left[c_{2}, c_{3}\right]$ and $\left(c_{3}, c_{4}\right]$ belongs to $M^{\prime}$. Otherwise, if $l^{\prime}$ has only a predecessor $\left[c_{1}, c_{2}\right)$ (respectively a successor $\left.\left(c_{3}, c_{4}\right]\right)$, then the interval $\left[c_{1}, c_{3}\right]$ obtained by fusion of $\left[c_{1}, c_{2}\right)$ and $\left[c_{2}, c_{3}\right]$ (respectively $\left[c_{2}, c_{4}\right]$ obtained by fusion of $\left[c_{2}, c_{3}\right]$ and $\left(c_{3}, c_{4}\right]$ ) belongs to $M^{\prime}$. Otherwise, $l^{\prime}$ itself belongs to $M^{\prime}$. Note that $M^{\prime}$ contains only intervals of $\hat{J}$ that are both left and right closed. Note also that every element $l$ that, seen over $\hat{J}$, is either left or right closed (or both) becomes part of some fusion which is contained in $M^{\prime}$.

Let us prove that the set $M \cup M^{\prime}$ satisfies the hypotheses of Lemma 8. It is a complete and scattered ordering with a least and a greatest element since it is a condensation of $L$. If $m=\left(c, c^{\prime}\right)$ belongs to $M$, it has a successor since the cut $c^{\prime}$ belongs to an interval $m^{\prime}$ which is left closed and belongs to $M^{\prime}$. Similarly, $m$ has a predecessor. Take $m<m^{\prime}$ two consecutive elements of $M \cup M^{\prime}$. If $m \in M$, we just proved that $m^{\prime} \in M^{\prime}$. If $m \in M^{\prime}$, it is impossible that $m^{\prime} \in M^{\prime}$ due to the possible configurations of consecutive elements in $L$. Hence, Lemma 8 holds and $M^{\prime}$ is isomorphic to $\hat{M}$.

For every $m=\left(c, c^{\prime}\right)$ in $M$, the label of the path $\gamma\left[c, c^{\prime}\right]$ belongs to (see (13), (15) and (16))

$$
X_{\tau, \tau^{\prime}}=\Gamma_{\tau, \tau^{\prime}}^{P} \cup \Delta_{\tau, \tau^{\prime}}^{P} \cup \nabla_{\tau, \tau^{\prime}}^{P} \quad \text { with } \quad \tau=\gamma(c) \rightarrow P \text { and } \tau^{\prime}=P \rightarrow \gamma\left(c^{\prime}\right) .
$$

Consider $m=\left[c, c^{\prime}\right]$ in $\hat{M}$. Let $y$ be the label of path $\gamma\left[c, c^{\prime}\right]$ (see (14), (17) and (18) and the definition of $\left.M^{\prime}\right)$. Suppose first that $c \neq c^{\prime}$, that is, $y \neq \varepsilon$. Let $\tau$ be the transition leaving $\gamma(c)$ and let $\tau^{\prime}$ be the transition entering $\gamma\left(c^{\prime}\right)$. The label $y$ belongs to

$$
\begin{aligned}
& Y_{\tau, \tau^{\prime}}=\bigcup_{\substack{\left(\tau_{1}^{\prime}, \tau_{1}\right) \in \mathcal{T}_{1} \\
\left(\tau_{2}^{\prime}, \tau_{2}\right) \in \mathcal{T}_{2}}} \Delta_{\tau, \tau_{1}^{\prime}}^{P} Z_{\tau_{1}, \tau_{2}^{\prime}} \nabla_{\tau_{2}, \tau^{\prime}}^{P} \quad \cup \quad \bigcup_{\left(\tau_{3}^{\prime}, \tau_{3}\right) \in \mathcal{T}_{3}} \Delta_{\tau, \tau_{3}^{\prime}}^{P} \nabla_{\tau_{3}, \tau^{\prime}}^{P} \\
& \cup \bigcup_{\left(\tau_{1}^{\prime}, \tau_{1}\right) \in \mathcal{T}_{1}} \Delta_{\tau, \tau_{1}^{\prime}}^{P} Z_{\tau_{1}, \tau^{\prime}} \quad \cup \quad \Delta_{\tau, \tau^{\prime}}^{P} \\
& \cup \bigcup_{\left(\tau_{2}^{\prime}, \tau_{2}\right) \in \mathcal{T}_{2}} Z_{\tau, \tau_{2}^{\prime}} \nabla_{\tau_{2}, \tau^{\prime}}^{P} \quad \cup \quad \nabla_{\tau, \tau^{\prime}}^{P} \\
& \cup \quad Z_{\tau, \tau^{\prime}}
\end{aligned}
$$

where the sets $\mathcal{T}_{1}, \mathcal{T}_{2}$ and $\mathcal{T}_{3}$ are defined by

$$
\begin{aligned}
& \mathcal{T}_{1}=\left\{\left(\tau^{\prime}, \tau\right) \mid \exists q \in P \quad \tau^{\prime}=P \rightarrow q, \tau \in \operatorname{Out}(q) \text { and } \tau \neq q \rightarrow P\right\} \\
& \mathcal{T}_{2}=\left\{\left(\tau^{\prime}, \tau\right) \mid \exists q \in P \quad \tau^{\prime} \in \operatorname{In}(q), \tau^{\prime} \neq P \rightarrow q \text { and } \tau=q \rightarrow P\right\} \\
& \mathcal{T}_{3}=\left\{\left(\tau^{\prime}, \tau\right) \mid \exists q \in P \quad \tau^{\prime}=P \rightarrow q \text { and } \tau=q \rightarrow P\right\} .
\end{aligned}
$$

Note that if $m$ is not the first element of $M \cup \hat{M}$, then $\tau$ belongs to $T_{1}=\{\tau \mid$ $\left.\exists \tau^{\prime}\left(\tau^{\prime}, \tau\right) \in \mathcal{T}_{1}\right\}$ and if $m$ is not the last element of $M \cup \hat{M}$, then $\tau^{\prime}$ belongs 
to $T_{2}^{\prime}=\left\{\tau^{\prime} \mid \exists \tau \quad\left(\tau^{\prime}, \tau\right) \in \mathcal{T}_{2}\right\}$. Otherwise, the set $Y_{\tau, \tau^{\prime}}$ is such that $\tau=\sigma$ or $\tau^{\prime}=\sigma^{\prime}$. Moreover, when $\tau=\sigma$ and $\tau^{\prime}=\sigma^{\prime}$, the definition of $Z_{\tau, \tau^{\prime}}$ given by Equation (14) must be slightly changed into $Z_{\sigma, \sigma^{\prime}}=\Gamma_{\sigma, \sigma^{\prime}}^{P}$ due to the content being equal to $P$.

Let us study the elements $m=\left[c, c^{\prime}\right]$ of $\hat{M}$ such that $c=c^{\prime}$. If $\hat{M}^{*}$ contains an element $[c, c]$, then $M \neq \varnothing$ and there are in $\mathcal{A}$ two transitions $P \rightarrow q$ and $q \rightarrow P$ for some state $q \in P$. Analogously, if the first element of $\hat{M}$ is equal to $[c, c]$, then $M \neq \varnothing$ and the first transition $\sigma$ is equal to $p \rightarrow P$ (similarly for the last element of $\hat{M}$ ).

We now come back to the label $x \in \Pi_{\sigma, \sigma^{\prime}}^{P}$ of the path $\gamma$. If $M=\varnothing$, then $\hat{M}$ is a singleton whose sole element is an interval $\left[c, c^{\prime}\right]$ with $c \neq c^{\prime}$. It follows that $x \in Y_{\sigma, \sigma^{\prime}}$. If $M \neq \varnothing$, we decompose $x$ thanks to the rational operation $\diamond$ used on $M \cup \hat{M}^{*}$. Different cases have to be considered depending on the transitions $\sigma$ and $\sigma^{\prime}$. Define the two sets $X$ and $Y$ by

$$
X=\bigcup_{\substack{q, q^{\prime} \in P \\ \tau=q \rightarrow P \\ \tau^{\prime}=P \rightarrow q^{\prime}}} X_{\tau, \tau^{\prime}} \quad \text { and } \quad Y=\bigcup_{\substack{\tau \in T_{1} \\ \tau^{\prime} \in T_{2}^{\prime}}} Y_{\tau, \tau^{\prime}}
$$

Add to $Y$ the empty word if there are in $\mathcal{A}$ two transitions $P \rightarrow q$ and $q \rightarrow P$ for some state $q \in P$. Define the set $Y_{\sigma}=\bigcup_{\tau^{\prime} \in T_{2}^{\prime}} Y_{\sigma, \tau^{\prime}}$ if $\sigma \neq p \rightarrow P$ and $Y_{\sigma}=\varepsilon$ if $\sigma=p \rightarrow P$. Define also the set $Y_{\sigma^{\prime}}=\bigcup_{\tau \in T_{1}} Y_{\tau, \sigma^{\prime}}$ if $\sigma^{\prime} \neq P \rightarrow p^{\prime}$ and $Y_{\sigma^{\prime}}=\varepsilon$ if $\sigma^{\prime}=P \rightarrow p^{\prime}$. Then the label $x$ belongs to $Y_{\sigma}(X \diamond Y) Y_{\sigma^{\prime}}$ showing the inclusion

$$
\Pi_{\sigma, \sigma^{\prime}}^{P} \subseteq Y_{\sigma, \sigma^{\prime}} \cup Y_{\sigma}(X \diamond Y) Y_{\sigma^{\prime}}
$$

It can be verified that the reverse inclusion holds as well. Clearly, the set $Y_{\sigma, \sigma^{\prime}}$ is included in $\Pi_{\sigma, \sigma^{\prime}}^{P}$. One checks that the right limit transitions $q \rightarrow P$ and the left limit transitions $P \rightarrow q^{\prime}$ involved in the operation $\diamond$ are well managed thanks to the conditions imposed by $T_{1}$ and $T_{2}^{\prime}$.

Therefore, $\Pi_{\sigma, \sigma^{\prime}}^{P}$ is expressed as a rational expression on the sets $\nabla_{\tau, \tau^{\prime}}^{P}, \Delta_{\tau, \tau^{\prime}}^{P}$, $\Gamma_{\tau, \tau^{\prime}}^{P}$ and $\Pi_{\tau, \tau^{\prime}}^{R}$ with $R \subsetneq P$. This completes the proof.

\section{Conclusion}

In this paper, we have introduced automata and rational expressions for words on linear orderings. We have proved that for words on countable scattered linear orderings, these two notions are equivalent. This result extends the usual Kleene's theorem for finite words.

We mention some open problems. A natural generalization of the result would be to remove the restrictions on the orderings, first considering words on countable linear orderings and then words on all linear orderings. Automata that we have introduced are suitable for all linear orderings. It seems however that new rational operations are then needed. An operation like the $\eta$-shuffle introduced in [15] is necessary. 
Automata on infinite words were introduced by Büchi to prove the decidability of the monadic second-order theory of the positive integers [7]. Since then, automata and logics have been shown to have strong connections [24]. The next step is to investigate the connections between logics and the automata that we have introduced. Such a study has to begin with the closure of the class of recognizable sets under the boolean operations. On the other hand, it is known that the monadic second-order theory of all linear orderings is undecidable [22] if the continuum hypothesis is assumed (see [21, p. 397] for a survey).

Since the submission of this article, new related results have been obtained. Carton and Rispal have proved that the class of recognizable sets introduced in this article is closed under complementation [20]. The emptiness problem for automata on scattered countable linear orderings has been shown to be decidable with a polynomial time complexity in [10]; the equivalence problem has been proved to be decidable in [6]. Very recently, Bès and Carton have shown that the equivalence between automata and rational expressions can be extended to all linear orderings if the $\eta$-shuffle is used [4].

\section{Acknowledgments}

We would like to thank the anonymous referees for their highly relevant comments and very helpful suggestions about the preliminary versions of this paper.

\section{References}

[1] E. Asarin, P. Caspi, and O. Maler. A Kleene theorem for timed automata. In Proceedings, Twelth Annual IEEE Symposium on Logic in Computer Science, pages 160-171, 1997.

[2] N. Bedon and O. Carton. An Eilenberg theorem for words on countable ordinals. In C. L. Lucchesi and A. V. Moura, editors, Latin'98: Theoretical Informatics, volume 1380 of Lect. Notes in Comput. Sci., pages 53-64. Springer-Verlag, 1998.

[3] B. Bérard and C. Picaronny. Accepting Zeno words without making time stand still. In Mathematical Foundations of Computer Science 1997, volume 1295 of Lect. Notes in Comput. Sci., pages 149-158, 1997.

[4] A. Bès and O. Carton. A Kleene theorem for languages of words indexed by linear orderings. In C. De Felice and A. Restivo, editors, DLT'2005, volume 3572 of Lect. Notes in Comput. Sci., pages 158-167. Springer-Verlag, 2005.

[5] V. Bruyère and O. Carton. Automata on linear orderings. In J. Sgall, A. Pultr, and P. Kolman, editors, MFCS'2001, volume 2136 of Lect. Notes in Comput. Sci., pages 236-247, 2001. 
[6] V. Bruyère, O. Carton, and G. Sénizergues. Tree automata and automata on linear orderings. In T. Harju and J. Karhumäki, editors, WORDS'2003, volume 27, pages 222-231. Turku Center for Computer Science, 2003.

[7] J. R. Büchi. Weak second-order arithmetic and finite automata. Z. Math. Logik und grundl. Math., 6:66-92, 1960.

[8] J. R. Büchi. On a decision method in the restricted second-order arithmetic. In Proc. Int. Congress Logic, Methodology and Philosophy of science, Berkeley 1960, pages 1-11. Stanford University Press, 1962.

[9] J. R. Büchi. Transfinite automata recursions and weak second order theory of ordinals. In Proc. Int. Congress Logic, Methodology, and Philosophy of Science, Jerusalem 1964, pages 2-23. North Holland, 1965.

[10] O. Carton. Accessibility in automata on scattered linear orderings. In K. Diks and W. Rytter, editors, MFCS'2002, volume 2420 of Lect. Notes in Comput. Sci., pages 155-164, 2002.

[11] Y. Choueka. Finite automata, definable sets, and regular expressions over $\omega^{n}$-tapes. J. Comput. System Sci., 17(1):81-97, 1978.

[12] B. Courcelle. Frontiers of infinite trees. RAIRO Theoretical informatics, 12(4):319-337, 1978.

[13] D. Girault-Beauquier. Bilimites de langages reconnaissables. Theoret. Comput. Sci., 33(2-3):335-342, 1984.

[14] M. R. Hansen, P. K. Pandya, and Z. Chaochen. Finite divergence. Theoret. Comput. Sci., 138(1):113-139, 1995.

[15] S. Heilbrunner. An algorithm for the solution of fixed-point equations for infinite words. RAIRO Theoretical informatics, 14(2):131-141, 1980.

[16] S. C. Kleene. Representation of events in nerve nets and finite automata. In C.E. Shannon, editor, Automata studies, pages 3-41. Princeton university Press, Princeton, 1956.

[17] M. Nivat and D. Perrin. Ensembles reconnaissables de mots bi-infinis. In Proceedings of the Fourteenth Annual ACM Symposium on Theory of Computing, pages 47-59, 1982.

[18] D. Perrin. Finite automata. In J. van Leeuwen, editor, Handbook of Theoretical Computer Science, volume B, chapter 1, pages 1-57. Elsevier, 1990.

[19] D. Perrin and J.-É. Pin. Infinite Words. Elsevier, 2004.

[20] C. Rispal and O. Carton. Complementation of rational sets on countable scattered linear orderings. J. Found. Comput. Sci., 16(4):767-786, 2005.

[21] J. G. Rosenstein. Linear Orderings. Academic Press, New York, 1982. 
[22] S. Shelah. The monadic theory of order. Annals of Mathematics, 102:379419, 1975.

[23] W. Thomas. On frontiers of regular sets. RAIRO Theoretical informatics, 20:371-381, 1986 .

[24] W. Thomas. Automata on infinite objects. In J. van Leeuwen, editor, Handbook of Theoretical Computer Science, volume B, chapter 4, pages 133-191. Elsevier, 1990.

[25] J. Wojciechowski. Finite automata on transfinite sequences and regular expressions. Fundamenta informatica, 8(3-4):379-396, 1985. 